\title{
Review
}

\section{Death after the Administration of COVID-19 Vaccines Approved by EMA: Has a Causal Relationship Been Demonstrated?}

\author{
Aniello Maiese ${ }^{1}$, Arianna Baronti ${ }^{1}$, Alice Chiara Manetti ${ }^{1}$ (D), Marco Di Paolo ${ }^{1}$, Emanuela Turillazzi ${ }^{1}$, \\ Paola Frati ${ }^{2}$ and Vittorio Fineschi ${ }^{2, *}$ (i)
}

1 Department of Surgical, Medical and Molecular Pathology and Critical Care Medicine, Institute of Legal Medicine, University of Pisa, Via Roma 55, 56126 Pisa, Italy; aniello.maiese@unipi.it (A.M.); arianna-baronti@virgilio.it (A.B.); a.manetti3@studenti.unipi.it (A.C.M.); marco.dipaolo@unipi.it (M.D.P.); emanuela.turillazzi@unipi.it (E.T.)

2 Department of Anatomical, Histological, Forensic and Orthopedic Sciences, Institute of Legal Medicine, Sapienza University of Rome, Viale Regina Elena 336, 00161 Rome, Italy; paola.frati@uniroma1.it

* Correspondence: vittorio.fineschi@uniroma1.it; Tel.: +39-0649912722

check for updates

Citation: Maiese, A.; Baronti, A.; Manetti, A.C.; Di Paolo, M.; Turillazzi, E.; Frati, P.; Fineschi, V. Death after the Administration of COVID-19 Vaccines Approved by EMA: Has a Causal Relationship Been Demonstrated? Vaccines 2022, 10, 308. https://doi.org/10.3390/ vaccines 10020308

Academic Editor: Vincenzo Baldo

Received: 1 January 2022

Accepted: 13 February 2022

Published: 16 February 2022

Publisher's Note: MDPI stays neutral with regard to jurisdictional claims in published maps and institutional affiliations.

Copyright: (C) 2022 by the authors. Licensee MDPI, Basel, Switzerland. This article is an open access article distributed under the terms and conditions of the Creative Commons Attribution (CC BY) license (https:// creativecommons.org/licenses/by/ $4.0 /)$.

\begin{abstract}
More than eight billion doses of COVID-19 vaccines have been administered globally so far and $44.29 \%$ of people are fully vaccinated. Pre-authorization clinical trials were carried out and the safety of vaccines is still continuously monitored through post-commercialization surveillance. However, some people are afraid of vaccine side effects, claiming they could lead to death, and hesitate to get vaccinated. Herein, a literature review of COVID-19-vaccine-related deaths has been carried out according to the PRISMA standards to understand if there is a causal relationship between vaccination and death and to highlight the real extent of such events. There have been 55 cases of death after COVID-19 vaccination reported and a causal relationship has been excluded in 17 cases. In the remaining cases, the causal link between the vaccine and the death was not specified (8) or considered possible (15), probable (1), or very probable/demonstrated (14). The causes of deaths among these cases were: vaccine-induced immune thrombotic thrombocytopenia (VITT) (32), myocarditis (3), ADEM (1), myocardial infarction (1), and rhabdomyolysis (1). In such cases, the demonstration of a causal relationship is not obvious, and more studies, especially with post-mortem investigations, are needed to deepen understanding of the possible pathophysiological mechanisms of fatal vaccine side effects. In any event, given the scarcity of fatal cases, the benefits of vaccination outweigh the risks and the scientific community needs to be cohesive in asserting that vaccination is fundamental to containing the spread of SARS-CoV-2.
\end{abstract}

Keywords: COVID-19; vaccine; death; side effects

\section{Introduction}

Since severe acute respiratory syndrome coronavirus 2 (SARS-CoV-2) has spread worldwide, the international scientific community has been focused on developing strategies to contain it $[1,2]$. Thanks to its efforts, to date, SARS-CoV-2 infection can be counteracted not only with simple preventative measures (e.g., face masks, handwashing, and physical distancing) but also with effective vaccines [3,4]. According to the Centers for Disease Control and Prevention (CDC), a vaccine is "a preparation that is used to stimulate the body's immune response against diseases" [5]. More than eight billion doses of COVID-19 vaccines have been administered globally so far, and $44.29 \%$ of people are fully vaccinated [6,7]. Regarding the European Union, about 660 million doses have been administered and more than 290 million $(298,845,193)$ people are fully vaccinated [8]. COVID-19 vaccines, due to the urgency of the global pandemic, were given emergency approval [9-12]. Clinical trials counting thousands of participants were carried out before the authorization $[13,14]$. Moreover, since the vaccines' administration to the population was authorized, 
their safety has been continuously monitored $[15,16]$. However, some people are afraid of vaccines' side effects, claiming they could lead to death, and hesitate to get vaccinated. Herein, we report a literature review concerning cases of death following these vaccines' administration to understand if there is a causal relationship and to highlight the real extent of such events.

\section{Materials and Methods}

The present systematic review was carried out according to the Preferred Reporting Items for Systematic Review (PRISMA) standards [17]. A systematic literature search and a critical review of the collected studies were conducted. An electronic search of PubMed, Science Direct Scopus, Google Scholar, and Excerpta Medica Database (EMBASE) from database inception to November 2021 was performed. The search terms were "COVID-19", "SARS-CoV-2", "vaccine", "vaccination", "death", and "autopsy" in the title, abstract, and keywords.

The bibliographies of all located papers were examined and cross-referenced to further identify relevant literature. A methodological appraisal of each study was conducted according to the PRISMA standards, including an evaluation of bias. The data collection process included study selection and data extraction. Two researchers (AB and ACM) independently examined the papers with titles or abstracts that appeared to be relevant and selected those that concerned cases of death after COVID-19 vaccination. Papers regarding anaphylaxis deaths were excluded, as were preprints and non-English articles. Disagreements concerning eligibility among the researchers were resolved by consensus. Data extraction was performed by two investigators ( $\mathrm{AB}$ and $\mathrm{ACM}$ ) and verified by two other investigators (AM and MDP). Two investigators drafted the manuscript (AB and $\mathrm{ACM}$ ) and other investigators (ET, PF, and VF) revised and finalized it. This study was exempt from institutional review board approval, as it did not involve human subjects.

\section{Results}

A review of the titles and abstracts as well as a manual search of the reference lists were carried out. The reference lists of all identified articles were reviewed to find missed literature. This search identified 407 articles, which were then screened to exclude duplicates. The resulting 389 reference lists were screened based on their abstract, which left 102 articles for further consideration. In addition, non-English papers were excluded, and the following inclusion criteria were used: (1) original research articles, (2) reviews and mini-reviews, and (3) case reports/series. These publications were carefully evaluated, taking into account the main aims of the review. Figure 1 illustrates our search strategy. This evaluation left 19 scientific papers comprising original research articles, case reports, and case series.

The 29 articles were then carefully evaluated to extract the useful information. Table 1 shows the results of our research and data extraction.

The sex and age of the subjects was not specified in all papers, therefore we used only papers in which these data were specified for the following calculations. The male/female ratio was close to one (1.04). The mean age was 52.74 years (range 22-91). The cases of death of people aged 50 years or younger were 21, while the cases of death of people older than 50 years were 26, with a ratio of 0.8 . Considering the types of COVID-19 vaccines authorized by EMA (BNT162b2, Comirnaty ${ }^{\circledR}$, BioNTech/Pfizer; mRNA1273, Spikevax ${ }^{\circledR}$, Moderna; adenovirus type 26 vector COVID-19 Vaccine Janssen, Janssen ${ }^{\circledR}$, Johnson\&Johnson; recombinant chimpanzee adenoviral vector vaccine ChAdOx1 nCoV-19, Vaxzevria ${ }^{\circledR}$, AstraZeneca), we found a total of 55 cases of death reported in the literature.

The causes of death were: vaccine-induced immune thrombotic thrombocytopenia (VITT) or, when VITT was not completely investigated, uncommonly located thrombosis associated with hemorrhages, in 32 cases (58.1\%); myocardial infarction and/or some kind of pre-existing cardiac changes in ten cases (18.2\%); myocarditis in three cases (5.4\%); pulmonary artery embolism (PAE) in three cases (5.4\%); acute disseminated encephalomyelitis 
(ADEM) in one case (1.8\%); massive cerebral hemorrhage not associated with thrombosis or auto-antibodies in one case $(1.8 \%)$; anaphylactic reaction to anesthetics associated with cerebral venous sinus thrombosis and anti-PF4 antibodies in one case (1.8\%); hyperglycemic coma in one case $(1.8 \%)$; hemorrhagic shock due to aortic dissection with rupture in one case $(1.8 \%)$; complications of rhabdomyolysis in one case $(1.8 \%)$. Figure 2 shows the distribution of the causes of death among the cases.
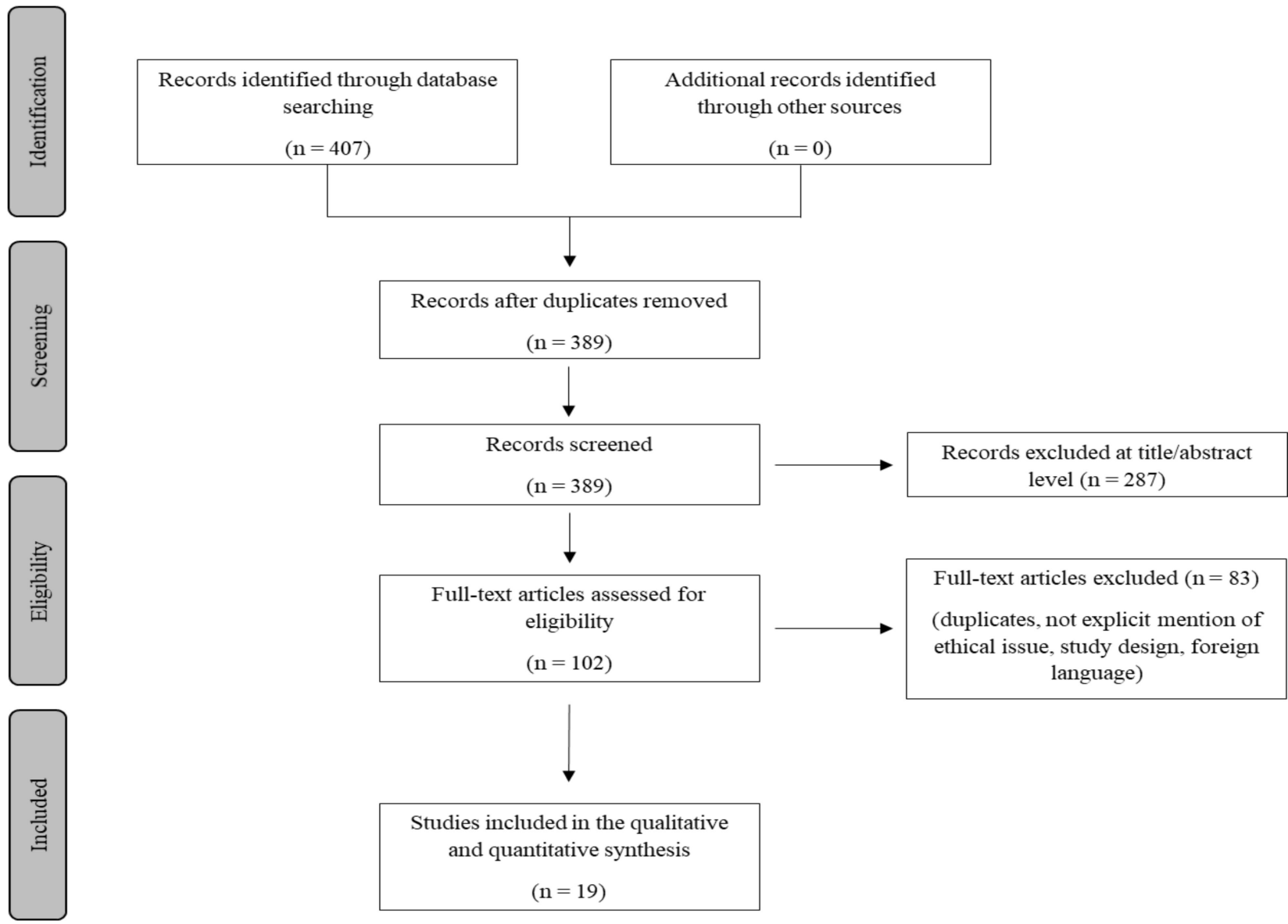

Figure 1. Methodology search strategy: we identified 389 articles after removing duplicates, the screening based on their abstracts left 102 studies, and after a careful evaluation based on the aims of this review 19 research articles were included. 
Table 1. A summary of the main information obtained from the results of our literature review. AAT indicates acute aortic thrombosis; ADEM, acute disseminated encephalomyelitis; AF, atrial fibrillation; CBN, contraction band necrosis; CeVD, cerebrovascular disease; $\mathrm{CI}$, cardiac insufficiency; CND, chronic neurologic disorder; COPD, chronic pulmonary disease; COVID-19, coronavirus disease 2019; CRP, C-reactive protein; CRF, chronic renal failure; CSF, cerebrospinal fluid; CVST, cerebral venous sinus thrombosis; DIC, disseminated intravascular coagulation; DM2, type 2 diabetes mellitus; DVT, deep vein thrombosis; FXIII, coagulation factor XIII; FVL, Factor V Leiden; HL, hyperlipidemia; HT, hypertension; IHD, ischemic heart disease; IL, interleukin; LN, lymph node; MI, myocardial infarction; MTHFR, methylenetetrahydrofolate reductase; NP, not performed; NS, not specified; PAD, peripheral artery disease; PAE, pulmonary embolism; PC, pseudomembranous colitis; PF4, platelet factor 4; PSC, primary sclerosing cholangitis; RA, rheumatoid arthritis; SplVT, splanchnic vein thrombosis; SVT, superficial vein thrombosis; VITT, vaccine-induced immune thrombotic thrombocytopenia. $\downarrow$ indicates reduction of levels.

\begin{tabular}{|c|c|c|c|c|c|c|c|c|c|c|c|}
\hline References & N. & Sex & Age (yrs) & $\begin{array}{l}\text { Pre-Existing } \\
\text { Conditions }\end{array}$ & $\begin{array}{l}\text { Type of } \\
\text { Vaccine }\end{array}$ & $\begin{array}{c}\text { Vax- } \\
\text { Symptoms } \\
\text { Interval }\end{array}$ & $\begin{array}{l}\text { Clinical Manifestations/ } \\
\text { Ante-Mortem Findings }\end{array}$ & $\begin{array}{c}\text { Post-Mortem } \\
\text { and/or } \\
\text { Autoptic Findings }\end{array}$ & $\begin{array}{l}\text { Cause of } \\
\text { Death }\end{array}$ & $\begin{array}{l}\text { Causal } \\
\text { Relation- } \\
\text { ship? }\end{array}$ & $\begin{array}{l}\text { Hypothetical } \\
\text { Pathophysiology }\end{array}$ \\
\hline $\begin{array}{c}\text { Ajmera } \\
2021 \text { [18] }\end{array}$ & 1 & $\mathrm{~F}$ & 85 & $\begin{array}{l}\text { RA, HL (on statin } \\
\text { therapy), asthma, } \\
\text { cerebrovascular } \\
\text { accident } 2 \text { months } \\
\text { earlier }\end{array}$ & $\begin{array}{l}\text { Spikevax } \\
\text { II dose }\end{array}$ & $\begin{array}{l}\text { Soon after } \\
(2 \text { days } \neq)\end{array}$ & $\begin{array}{l}\text { Rhabdomyolysis } \\
\text { complicated by } \\
\text { pneumonia during } \\
\text { hospitalization }\end{array}$ & NP & NS & NS & $\begin{array}{l}\text { Immune- } \\
\text { mediated }\end{array}$ \\
\hline $\begin{array}{l}\text { Bjørnstad- } \\
\text { Tuveng } \\
\text { et al. } \\
2021[19]\end{array}$ & 1 & $\mathrm{~F}$ & $30 \mathrm{~s}$ & $\begin{array}{l}\text { Preeclampsia and } \\
\text { huge bleeding } \\
\text { during childbirth } \\
11 \text { months before }\end{array}$ & $\begin{array}{l}\text { Vaxzevria } \\
\text { unknown } \\
\text { dose }\end{array}$ & $\begin{array}{l}7 \text { days }(10 \\
\text { days } \neq)\end{array}$ & $\begin{array}{c}\downarrow \text { PLTs + intracerebral } \\
\text { hemorrhage }\end{array}$ & $\begin{array}{l}\text { CVST (small } \\
\text { thrombi) + anti-PF4 } \\
\text { antibodies }\end{array}$ & VITT & Probable & $\begin{array}{l}\text { Immune- } \\
\text { mediated }\end{array}$ \\
\hline $\begin{array}{l}\text { Castelli } \\
\text { et al. } \\
2021[20]\end{array}$ & 1 & $\mathrm{M}$ & 50 & $\begin{array}{c}\text { Heterozygous } \\
\text { MTHFR mutation } \\
(\mathrm{C} 677 \mathrm{~T})\end{array}$ & $\begin{array}{l}\text { Vaxzevria } \\
\text { I dose }\end{array}$ & $\begin{array}{l}7 \text { days }(11 \\
\text { days } \neq)\end{array}$ & $\begin{array}{c}\downarrow \text { PLTs, fibrinogen, FXIII + } \\
\text { CVST + intracerebral } \\
\text { hemorrhage }\end{array}$ & NP & $\begin{array}{c}\text { CVST + } \\
\text { intracerebral } \\
\text { hemorrhage }\end{array}$ & NS & NS \\
\hline $\begin{array}{l}\text { Choi et al. } \\
2021[21]\end{array}$ & 1 & $\mathrm{M}$ & 22 & $\begin{array}{l}\text { Elevated blood } \\
\text { pressure in two } \\
\text { previous } \\
\text { measurements }\end{array}$ & $\begin{array}{l}\text { Comirnaty } \\
\text { I dose }\end{array}$ & 5 days & $\begin{array}{c}\text { Chest pain, VF the day } \\
\text { after }\end{array}$ & $\begin{array}{c}\text { Atrial myocarditis } \\
\text { histological features } \\
\text { + non-inflammatory } \\
\text { single-cell necrosis + } \\
\text { diffuse CBN }\end{array}$ & Myocarditis & Possible & $\begin{array}{c}\text { Immune- } \\
\text { mediated } \\
\text { (cytokine- } \\
\text { mediated or } \\
\text { histiocyte-linked } \\
\text { immunologic } \\
\text { injury) }\end{array}$ \\
\hline $\begin{array}{l}\text { D'Agostino } \\
\text { et al. } \\
2021[22]\end{array}$ & 1 & $\mathrm{~F}$ & 54 & Meniere's disease & $\begin{array}{l}\text { Vaxzevria } \\
\text { unknown } \\
\text { dose }\end{array}$ & 12 days $\neq$ & $\begin{array}{l}\text { CVST and DIC (arterial } \\
\text { and venous) }\end{array}$ & NP & $\begin{array}{l}\text { CVST and } \\
\text { DIC }\end{array}$ & Possible & NS \\
\hline
\end{tabular}


Table 1. Cont.

\begin{tabular}{|c|c|c|c|c|c|c|c|c|c|c|c|}
\hline References & N. & Sex & Age (yrs) & $\begin{array}{l}\text { Pre-Existing } \\
\text { Conditions }\end{array}$ & $\begin{array}{l}\text { Type of } \\
\text { Vaccine }\end{array}$ & $\begin{array}{c}\text { Vax- } \\
\text { Symptoms } \\
\text { Interval }\end{array}$ & $\begin{array}{l}\text { Clinical Manifestations/ } \\
\text { Ante-Mortem Findings }\end{array}$ & $\begin{array}{c}\text { Post-Mortem } \\
\text { and/or } \\
\text { Autoptic Findings }\end{array}$ & $\begin{array}{l}\text { Cause of } \\
\text { Death }\end{array}$ & $\begin{array}{l}\text { Causal } \\
\text { Relation- } \\
\text { ship? }\end{array}$ & $\begin{array}{c}\text { Hypothetical } \\
\text { Pathophysiology }\end{array}$ \\
\hline \multirow{3}{*}{$\begin{array}{c}\text { Edler } \\
\text { et al. } \\
2021[23]\end{array}$} & \multirow{3}{*}{3} & $\mathrm{~F}$ & Elderly* & $\begin{array}{c}\text { IHD, CI, HT, } \\
\text { dementia, } \\
\text { hyperthyroidism, } \\
\text { pulmonary } \\
\text { emphysema, PC }\end{array}$ & $\begin{array}{l}\text { Comirnaty } \\
\text { I dose }\end{array}$ & $\begin{array}{c}3 \text { days }(5 \\
\left.\text { days }{ }^{¥}\right)\end{array}$ & $\begin{array}{l}\text { Fever three days after } \\
\text { vaccination, then } \\
\text { deteriorated and died }\end{array}$ & $\begin{array}{c}\text { Leg DVT }+ \text { PAE }+ \\
\text { cerebral infarct }+\uparrow \\
\text { CRP and IL-6 } \\
\text { (consistent with PC) }\end{array}$ & PAE & \multirow{3}{*}{ No } & \multirow{3}{*}{-} \\
\hline & & $\mathrm{M}$ & Elderly* & $\begin{array}{c}\text { CRF, anemia, AF, } \\
\text { PAE, HT, PAD, } \\
\text { CeVD, RA, } \\
\text { previous prostate } \\
\text { carcinoma, chronic } \\
\text { pancreatitis }\end{array}$ & $\begin{array}{l}\text { Comirnaty } \\
\text { I dose }\end{array}$ & 7 days & $\begin{array}{l}\text { COVID-19 pneumonia } \\
\text { (positive nasopharyngeal } \\
\text { swab } 12 \text { days after } \\
\text { vaccination) }\end{array}$ & $\begin{array}{l}\text { Lung histology } \\
\text { consistent with } \\
\text { COVID-19 } \\
\text { pneumonia }\end{array}$ & COVID-19 & & \\
\hline & & $\mathrm{M}$ & Elderly* & $\begin{array}{l}\text { HT, IHD, DM2, } \\
\text { CeVD, dementia, } \\
\text { COPD, CRF }\end{array}$ & $\begin{array}{l}\text { Comirnaty } \\
\text { I dose }\end{array}$ & 2 days $¥$ & $\begin{array}{l}\text { Unknown (found dead at } \\
\text { home) }\end{array}$ & $\begin{array}{c}\text { Peripheral PAE } \\
\text { (mostly organized, } \\
\text { some fresh) + } \\
\text { swollen axillary LNs } \\
\text { (near injection site) }\end{array}$ & $\begin{array}{l}\text { Recurrent MI } \\
+ \text { IHD }\end{array}$ & & \\
\hline $\begin{array}{l}\text { Franchini } \\
\text { et al. } \\
2021[24]\end{array}$ & 1 & $\mathrm{M}$ & 50 & $\begin{array}{l}\text { Heterozygous } \\
\text { MTHFR mutation } \\
\text { (C677T), folate } \\
\text { deficiency }\end{array}$ & $\begin{array}{l}\text { Vaxzevria } \\
\text { I dose }\end{array}$ & 7 days & $\begin{array}{c}\downarrow \text { PLTs, fibrinogen, FXIII + } \\
\text { anti-PF4 antibodies }+ \\
\text { CVST + intracerebral } \\
\text { hemorrhage }\end{array}$ & NP & $\begin{array}{c}\text { CVST + } \\
\text { intracerebral } \\
\text { hemorrhage }\end{array}$ & NS & $\begin{array}{c}\text { Immune- } \\
\text { mediated } \\
\text { (autoimmune or } \\
\text { protein } \\
\text { spike-mediated) }\end{array}$ \\
\hline \multirow{3}{*}{$\begin{array}{l}\text { Greinacher } \\
\text { et al. } \\
2021 \S \\
{[25]}\end{array}$} & \multirow{3}{*}{$6^{+}$} & $\mathrm{F}$ & 49 & None & $\begin{array}{l}\text { Vaxzevria } \\
\text { I dose }\end{array}$ & 5 days & $\begin{array}{c}\downarrow \text { PLTs, fibrinogen }+ \\
\text { anti-PF4 antibodies }+ \\
\text { SplVT }+ \text { peripheral PAE }\end{array}$ & CVST & VITT & \multirow{3}{*}{ Yes } & \multirow{3}{*}{$\begin{array}{c}\text { Immune- } \\
\text { mediated } \\
\text { (autoantibodies or } \\
\text { vaccine-induced } \\
\text { antibodies that } \\
\text { cross-react with } \\
\text { PF4 and PLTs) }\end{array}$} \\
\hline & & - & - & $\mathrm{CND}$ & $\begin{array}{l}\text { Vaxzevria } \\
\text { I dose }\end{array}$ & 7 days & $\begin{array}{c}\downarrow \text { PLTs + anti-PF4 } \\
\text { antibodies + CVST }\end{array}$ & NS & VITT & & \\
\hline & & - & - & None & $\begin{array}{l}\text { Vaxzevria } \\
\text { I dose }\end{array}$ & 8 days & $\begin{array}{l}\downarrow \text { PLTs + anti-PF4 } \\
\text { antibodies + CVST }\end{array}$ & $\begin{array}{l}\text { Widespread } \\
\text { microvascular } \\
\text { thrombosis }\end{array}$ & VITT & & \\
\hline
\end{tabular}


Table 1. Cont.

\begin{tabular}{|c|c|c|c|c|c|c|c|c|c|c|c|}
\hline References & N. & Sex & Age (yrs) & $\begin{array}{l}\text { Pre-Existing } \\
\text { Conditions }\end{array}$ & $\begin{array}{l}\text { Type of } \\
\text { Vaccine }\end{array}$ & $\begin{array}{c}\text { Vax- } \\
\text { Symptoms } \\
\text { Interval }\end{array}$ & $\begin{array}{l}\text { Clinical Manifestations/ } \\
\text { Ante-Mortem Findings }\end{array}$ & $\begin{array}{c}\text { Post-Mortem } \\
\text { and/or } \\
\text { Autoptic Findings }\end{array}$ & $\begin{array}{l}\text { Cause of } \\
\text { Death }\end{array}$ & $\begin{array}{l}\text { Causal } \\
\text { Relation- } \\
\text { ship? }\end{array}$ & $\begin{array}{c}\text { Hypothetical } \\
\text { Pathophysiology }\end{array}$ \\
\hline & & - & - & None & $\begin{array}{l}\text { Vaxzevria } \\
\text { I dose }\end{array}$ & 16 days & $\begin{array}{c}\downarrow \text { PLTs, fibrinogen }+ \\
\text { anti-PF4 antibodies }+ \\
\text { CVST }\end{array}$ & $\begin{array}{l}\text { Multiple organ } \\
\text { thrombi }\end{array}$ & VITT & & \\
\hline & & - & - & None & $\begin{array}{l}\text { Vaxzevria } \\
\text { I dose }\end{array}$ & 11 days & $\begin{array}{c}\downarrow \text { PLTs, fibrinogen }+ \\
\text { anti-PF4 antibodies }+ \\
\text { CVST }+ \text { SVT }\end{array}$ & NS & VITT & & \\
\hline & & - & - & Unknown & $\begin{array}{l}\text { Vaxzevria } \\
\text { I dose }\end{array}$ & 12 days & Found dead & $\begin{array}{c}\text { Cerebral } \\
\text { hemorrhage }\end{array}$ & VITT & & \\
\hline $\begin{array}{l}\text { Jamme } \\
\text { et al. } \\
2021[26]\end{array}$ & 1 & $\mathrm{~F}$ & 69 & HT & $\begin{array}{l}\text { Vaxzevria } \\
\text { I dose }\end{array}$ & 11 days & $\begin{array}{c}\downarrow \text { PLTs + anti-PF4 } \\
\text { antibodies + CVST + } \\
\text { intracerebral hemorrhage } \\
+ \text { segmentary PAE }\end{array}$ & $\mathrm{NP}$ & $\begin{array}{c}\text { CVST + } \\
\text { intracerebral } \\
\text { hemorrhage }\end{array}$ & NS & NS \\
\hline \multirow{2}{*}{$\begin{array}{l}\text { Mehta } \\
\text { et al. } \\
2021[27]\end{array}$} & \multirow[b]{2}{*}{2} & M & 32 & None & $\begin{array}{l}\text { Vaxzevria } \\
\text { I dose }\end{array}$ & 9 days & $\begin{array}{c}\downarrow \text { PLTs, fibrinogen }+ \text { CVST } \\
+ \text { intracerebral } \\
\text { hemorrhage }\end{array}$ & $\mathrm{NP}$ & $\begin{array}{c}\text { CVST + } \\
\text { intracerebral } \\
\text { hemorrhage }\end{array}$ & \multirow[b]{2}{*}{ NS } & \multirow[b]{2}{*}{$\begin{array}{l}\text { Immune- } \\
\text { mediated }\end{array}$} \\
\hline & & M & 25 & $\begin{array}{c}\text { PSC, migraines, } \\
\text { heterozygous FVL } \\
\text { mutation } \\
\text { (c.1601G>A) }\end{array}$ & $\begin{array}{l}\text { Vaxzevria } \\
\text { I dose }\end{array}$ & 6 days & $\begin{array}{c}\downarrow \text { PLTs, fibrinogen }+ \\
\text { anti-PF4 antibodies }+ \\
\text { CVST + intracerebral } \\
\text { hemorrhage }\end{array}$ & $\mathrm{NP}$ & $\begin{array}{c}\text { CVST + } \\
\text { intracerebral } \\
\text { hemorrhage }\end{array}$ & & \\
\hline $\begin{array}{l}\text { Permezel } \\
\text { et al. } \\
2021[28]\end{array}$ & 1 & M & 63 & $\mathrm{DM} 2, \mathrm{IHD}, \mathrm{AF}$ & $\begin{array}{l}\text { Vaxzevria } \\
\text { I dose }\end{array}$ & 12 days $\neq$ & ADEM & $\begin{array}{c}\text { Diffuse acute } \\
\text { demyelination } \\
\text { (perivenular) with } \\
\text { sparse lymphocytes }\end{array}$ & ADEM & NS & NS \\
\hline $\begin{array}{l}\text { Pomara } \\
\text { et al. } \\
2021[29]\end{array}$ & 2 & M & 50 & None & $\begin{array}{l}\text { Vaxzevria } \\
\text { I dose }\end{array}$ & 10 days $\neq$ & $\begin{array}{c}\downarrow \text { PLTs, fibrinogen }+ \\
\text { anti-PF4 antibodies }+ \\
\text { SplVT + intracerebral } \\
\text { hemorrhage }\end{array}$ & $\begin{array}{c}\text { Multi-organ small } \\
\text { and medium vessels } \\
\text { thrombi + } \\
\text { multi-organ } \\
\text { endothelial } \\
\text { activation }\end{array}$ & VITT & Yes & NS \\
\hline
\end{tabular}


Table 1. Cont.

\begin{tabular}{|c|c|c|c|c|c|c|c|c|c|c|c|}
\hline References & N. & Sex & Age (yrs) & $\begin{array}{l}\text { Pre-Existing } \\
\text { Conditions }\end{array}$ & $\begin{array}{l}\text { Type of } \\
\text { Vaccine }\end{array}$ & $\begin{array}{c}\text { Vax- } \\
\text { Symptoms } \\
\text { Interval }\end{array}$ & $\begin{array}{l}\text { Clinical Manifestations/ } \\
\text { Ante-Mortem Findings }\end{array}$ & $\begin{array}{c}\text { Post-Mortem } \\
\text { and/or } \\
\text { Autoptic Findings }\end{array}$ & $\begin{array}{l}\text { Cause of } \\
\text { Death }\end{array}$ & $\begin{array}{l}\text { Causal } \\
\text { Relation- } \\
\text { ship? }\end{array}$ & $\begin{array}{c}\text { Hypothetical } \\
\text { Pathophysiology }\end{array}$ \\
\hline & & $\mathrm{F}$ & 37 & None & $\begin{array}{l}\text { Vaxzevria } \\
\text { I dose }\end{array}$ & 10 days $\neq$ & $\begin{array}{c}\downarrow \text { PLTs, fibrinogen }+ \\
\text { anti-PF4 antibodies }+ \\
\text { CVST }+ \text { intracerebral } \\
\text { hemorrhage }\end{array}$ & $\begin{array}{c}\text { Massive upper limb } \\
\text { DVT + feet SVT + } \\
\text { multi-organ small } \\
\text { and medium vessels } \\
\text { thrombi + } \\
\text { multi-organ } \\
\text { endothelial } \\
\text { activation }\end{array}$ & VITT & & \\
\hline $\begin{array}{l}\text { Rodriguez } \\
\text { et al. } 2021 \\
\text { [30] }\end{array}$ & 1 & $\mathrm{~F}$ & 37 & None & Janssen & 7 days & $\begin{array}{c}\downarrow \text { PLTs, fibrinogen }+ \\
\text { anti-PF4 antibodies + } \\
\text { DVT + CVST + } \\
\text { intracerebral hemorrhage }\end{array}$ & NP & VITT & Yes & $\begin{array}{l}\text { Immune- } \\
\text { mediated } \\
\text { (exaggerated } \\
\text { response to the } \\
\text { vector) }\end{array}$ \\
\hline $\begin{array}{l}\text { Sangli et al. } \\
2021[31]\end{array}$ & 1 & M & 65 & HT, HP & $\begin{array}{l}\text { Spikevax } \\
\text { II dose }\end{array}$ & $\begin{array}{l}10 \text { days }(17 \\
\text { days } \neq)\end{array}$ & $\begin{array}{c}\downarrow \text { PLTs + anti-PF4 } \\
\text { antibodies + bilateral } \\
\text { lower extremities DVT + } \\
\text { acute bilateral PAE + } \\
\text { acute gluteal hematoma + } \\
\text { CVST + upper extremity } \\
\text { DVT + lower extremities } \\
\text { compartment syndrome + } \\
\text { S. aureus sepsis }\end{array}$ & $\mathrm{NP}$ & $\begin{array}{c}\text { VITT } \\
\text { (complicated } \\
\text { by sepsis) }\end{array}$ & NS & NS \\
\hline \multirow{3}{*}{$\begin{array}{l}\text { Schneider } \\
\text { et al. } 2021 \\
{[32]}\end{array}$} & \multirow{3}{*}{18} & $\mathrm{M}$ & 82 & NS & $\begin{array}{l}\text { Spikevax } \\
\text { I dose }\end{array}$ & 1 day ${ }^{¥}$ & Unknown (died at home) & $\begin{array}{l}\text { Coronary sclerosis + } \\
\text { cardiac hypertrophy } \\
\text { + MI scars }\end{array}$ & $\begin{array}{l}\text { Pre-existing } \\
\text { cardiac } \\
\text { changes }\end{array}$ & \multirow[b]{2}{*}{ No } & \multirow{2}{*}{ - } \\
\hline & & $\mathrm{F}$ & 91 & NS & $\begin{array}{l}\text { Spikevax } \\
\text { I dose }\end{array}$ & 1 day $¥$ & Unknown (died at home) & $\begin{array}{l}\text { Coronary sclerosis + } \\
\text { cardiac hypertrophy } \\
\text { + MI scars }\end{array}$ & $\begin{array}{l}\text { Pre-existing } \\
\text { cardiac } \\
\text { changes }\end{array}$ & & \\
\hline & & $\mathrm{F}$ & 32 & NS & $\begin{array}{l}\text { Vaxzevria } \\
\text { I dose }\end{array}$ & 12 days $¥$ & Unknown (died at home) & $\begin{array}{c}\text { Massive cerebral } \\
\text { hemorrhage }+ \\
\text { anti-PF4 antibody } \\
\text { (VITT) }\end{array}$ & $\begin{array}{c}\text { Massive } \\
\text { cerebral } \\
\text { hemorrhage }\end{array}$ & $\begin{array}{c}\text { Very } \\
\text { probable }\end{array}$ & $\begin{array}{l}\text { Immune- } \\
\text { mediated }\end{array}$ \\
\hline
\end{tabular}


Table 1. Cont.

\begin{tabular}{|c|c|c|c|c|c|c|c|c|c|c|c|}
\hline References & N. & Sex & Age (yrs) & $\begin{array}{l}\text { Pre-Existing } \\
\text { Conditions }\end{array}$ & $\begin{array}{l}\text { Type of } \\
\text { Vaccine }\end{array}$ & $\begin{array}{c}\text { Vax- } \\
\text { Symptoms } \\
\text { Interval }\end{array}$ & $\begin{array}{l}\text { Clinical Manifestations/ } \\
\text { Ante-Mortem Findings }\end{array}$ & $\begin{array}{c}\text { Post-Mortem } \\
\text { and/or } \\
\text { Autoptic Findings }\end{array}$ & $\begin{array}{l}\text { Cause of } \\
\text { Death }\end{array}$ & $\begin{array}{l}\text { Causal } \\
\text { Relation- } \\
\text { ship? }\end{array}$ & $\begin{array}{c}\text { Hypothetical } \\
\text { Pathophysiology }\end{array}$ \\
\hline & & $\mathrm{F}$ & 34 & Obesity & $\begin{array}{l}\text { Vaxzevria } \\
\text { I dose }\end{array}$ & 1 day ${ }^{¥}$ & Unknown (died at home) & $\begin{array}{c}\text { Cardiac } \\
\text { hypertrophy + MI } \\
\text { scars + fresh MI }\end{array}$ & Recurrent MI & \multirow[t]{2}{*}{ No } & \multirow[t]{2}{*}{-} \\
\hline & & $\mathrm{F}$ & 48 & NS & $\begin{array}{l}\text { Vaxzevria } \\
\text { I dose }\end{array}$ & 10 days ${ }^{*}$ & $\begin{array}{l}\text { Unknown (died at the } \\
\text { workplace) }\end{array}$ & $\begin{array}{l}\text { Aortic dissection } \\
\text { with rupture }\end{array}$ & $\begin{array}{c}\text { Bleeding } \\
\text { aorta }\end{array}$ & & \\
\hline & & $\mathrm{M}$ & 65 & NS & $\begin{array}{l}\text { Comirnaty } \\
\text { I dose }\end{array}$ & $11 \mathrm{~h}^{¥}$ & Unknown (died at home) & $\begin{array}{c}\text { Myocarditis + } \\
\text { coronary sclerosis + } \\
\text { cardiac hypertrophy } \\
\text { + MI scars }\end{array}$ & Myocarditis & Possible & $\begin{array}{l}\text { Immune- } \\
\text { mediated }\end{array}$ \\
\hline & & $\mathrm{M}$ & 71 & NS & $\begin{array}{l}\text { Comirnaty } \\
\text { I dose }\end{array}$ & 1 day ${ }^{¥}$ & Unknown (died at home) & $\begin{array}{c}\text { Cardiac } \\
\text { hypertrophy }+ \\
\text { coronary sclerosis }\end{array}$ & $\begin{array}{c}\text { Pre-existing } \\
\text { cardiac } \\
\text { changes }\end{array}$ & & \\
\hline & & $\mathrm{F}$ & 57 & NS & $\begin{array}{l}\text { Spikevax } \\
\text { II dose }\end{array}$ & 6 days $¥$ & Unknown (died at home) & $\begin{array}{l}\text { Coronary sclerosis }+ \\
\text { fatty liver }+ \text { high } \\
\text { levels of glucose and } \\
\text { lactate (in CSF and } \\
\text { aqueous humor) }\end{array}$ & $\begin{array}{l}\text { Hyperglycemic } \\
\text { coma }\end{array}$ & & \\
\hline & & $\mathrm{M}$ & 63 & NS & $\begin{array}{l}\text { Vaxzevria } \\
\text { I dose }\end{array}$ & 14 days $¥$ & Unknown (died at home) & $\begin{array}{c}\text { Coronary sclerosis + } \\
\text { cardiac hypertrophy } \\
+ \text { MI scars + liver } \\
\text { cirrhosis }\end{array}$ & $\begin{array}{c}\text { Pre-existing } \\
\text { cardiac } \\
\text { changes }\end{array}$ & No & - \\
\hline & & $\mathrm{M}$ & 61 & NS & $\begin{array}{l}\text { Vaxzevria } \\
\text { I dose }\end{array}$ & day $¥$ & Unknown (died at home) & $\begin{array}{c}\text { Coronary sclerosis }+ \\
\text { cardiac } \\
\text { hypertrophy }\end{array}$ & $\begin{array}{l}\text { Pre-existing } \\
\text { cardiac } \\
\text { changes }\end{array}$ & & \\
\hline & & $\mathrm{M}$ & 71 & NS & $\begin{array}{l}\text { Vaxzevria } \\
\text { unknown } \\
\text { dose }\end{array}$ & 10 days $¥$ & NS & $\begin{array}{c}\text { DVT + PAE + } \\
\text { coronary sclerosis + } \\
\text { cardiac hypertrophy } \\
\text { + MI scars } \\
\text { (VITT-diagnostics } \\
\text { negative) }\end{array}$ & PAE & & \\
\hline
\end{tabular}


Table 1. Cont.

\begin{tabular}{|c|c|c|c|c|c|c|c|c|c|c|c|}
\hline References & N. & Se & Age (yrs) & $\begin{array}{l}\text { Pre-Existing } \\
\text { Conditions }\end{array}$ & $\begin{array}{l}\text { Type of } \\
\text { Vaccine }\end{array}$ & $\begin{array}{c}\text { Vax- } \\
\text { Symptoms } \\
\text { Interval }\end{array}$ & $\begin{array}{l}\text { Clinical Manifestations/ } \\
\text { Ante-Mortem Findings }\end{array}$ & $\begin{array}{c}\text { Post-Mortem } \\
\text { and/or } \\
\text { Autoptic Findings }\end{array}$ & $\begin{array}{l}\text { Cause of } \\
\text { Death }\end{array}$ & $\begin{array}{l}\text { Causal } \\
\text { Relation- } \\
\text { ship? }\end{array}$ & $\begin{array}{c}\text { Hypothetical } \\
\text { Pathophysiology }\end{array}$ \\
\hline & & $\mathrm{F}$ & 38 & NS & $\begin{array}{l}\text { Vaxzevria } \\
\text { II dose }\end{array}$ & 8 days $¥$ & $\begin{array}{c}\text { Anaphylactic shock } \\
\text { during narcosis induction }\end{array}$ & $\begin{array}{c}\text { CVST + multiple } \\
\text { fresh thrombi }+ \\
\text { cardiac hypertrophy } \\
+ \text { MI + hypoxic brain } \\
\text { changes, anti-PF4 } \\
\text { antibodies }\end{array}$ & $\begin{array}{l}\text { Anaphylactic } \\
\text { reaction to } \\
\text { anesthetics } \\
\text { (thrombi } \\
\text { formed after } \\
\text { the brain } \\
\text { damage due } \\
\text { to the shock) }\end{array}$ & Improbable & $\begin{array}{l}\text { Immune- } \\
\text { mediated }\end{array}$ \\
\hline & & $\mathrm{F}$ & 72 & NS & $\begin{array}{l}\text { Comirnaty } \\
\text { I dose }\end{array}$ & 12 days $¥$ & Unknown (died at home) & $\begin{array}{c}\text { Massive cerebral } \\
\text { hemorrhage }+ \\
\text { coronary sclerosis }+ \\
\text { cardiac hypertrophy } \\
\text { (VITT diagnostics } \\
\text { negative) }\end{array}$ & $\begin{array}{c}\text { Massive } \\
\text { cerebral } \\
\text { hemorrhage }\end{array}$ & No & - \\
\hline & & $\mathrm{F}$ & 65 & NS & $\begin{array}{l}\text { Vaxzevria } \\
\text { I dose }\end{array}$ & 10 days ${ }^{¥}$ & $\begin{array}{l}\text { CVST + cerebral } \\
\text { hemorrhages }\end{array}$ & $\begin{array}{c}\text { CVST + cerebral } \\
\text { hemorrhages + } \\
\text { coronary sclerosis + } \\
\text { anti-PF4 antibodies }\end{array}$ & VITT & $\begin{array}{c}\text { Very } \\
\text { probable }\end{array}$ & $\begin{array}{l}\text { Immune- } \\
\text { mediated }\end{array}$ \\
\hline & & M & 79 & NS & $\begin{array}{l}\text { Comirnaty } \\
\text { II dose }\end{array}$ & 6 days $¥$ & Unknown (died at home) & $\begin{array}{c}\text { DVT + massive PAE } \\
\text { + coronary sclerosis } \\
\text { + pericarditis + } \\
\text { chronic pulmonary } \\
\text { emphysema (VITT } \\
\text { diagnostics } \\
\text { negative) }\end{array}$ & PAE & \multirow[t]{2}{*}{ No } & \multirow[t]{2}{*}{-} \\
\hline & & M & 57 & NS & $\begin{array}{l}\text { Vaxzevria } \\
\text { unknown } \\
\text { dose }\end{array}$ & 2 days $¥$ & NS & $\begin{array}{c}\text { Coronary sclerosis + } \\
\text { cardiac } \\
\text { hypertrophy + MI } \\
\text { scars + fresh MI }\end{array}$ & Recurrent MI & & \\
\hline
\end{tabular}


Table 1. Cont.

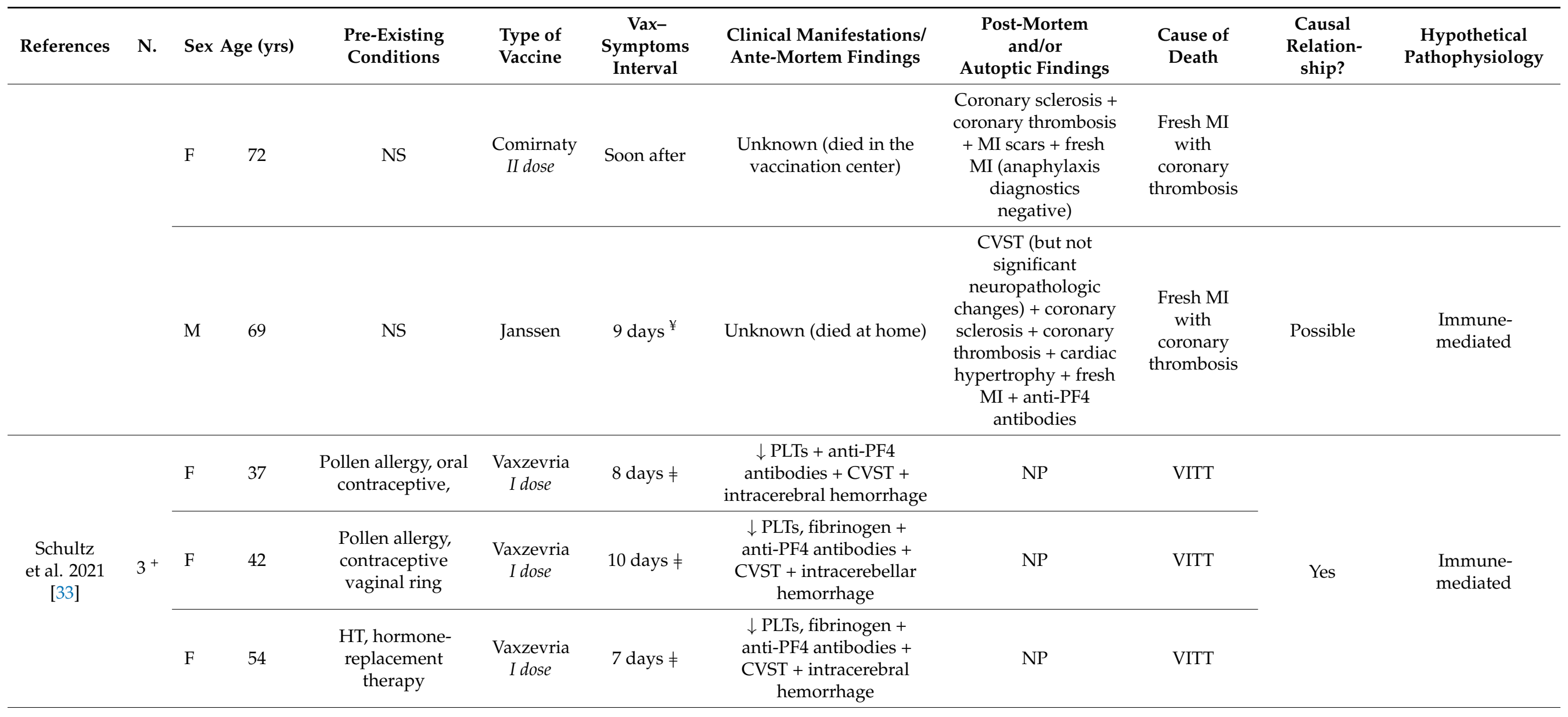


Table 1. Cont.

\begin{tabular}{|c|c|c|c|c|c|c|c|c|c|c|c|}
\hline References & N. & Sex & Age (yrs) & $\begin{array}{l}\text { Pre-Existing } \\
\text { Conditions }\end{array}$ & $\begin{array}{l}\text { Type of } \\
\text { Vaccine }\end{array}$ & $\begin{array}{l}\text { Vax- } \\
\text { Symptoms } \\
\text { Interval }\end{array}$ & $\begin{array}{l}\text { Clinical Manifestations/ } \\
\text { Ante-Mortem Findings }\end{array}$ & $\begin{array}{c}\text { Post-Mortem } \\
\text { and/or } \\
\text { Autoptic Findings }\end{array}$ & $\begin{array}{l}\text { Cause of } \\
\text { Death }\end{array}$ & $\begin{array}{l}\text { Causal } \\
\text { Relation- } \\
\text { ship? }\end{array}$ & $\begin{array}{l}\text { Hypothetical } \\
\text { Pathophysiology }\end{array}$ \\
\hline \multirow{6}{*}{$\begin{array}{l}\text { Scully et al. } \\
2021 \text { [34] }\end{array}$} & \multirow{6}{*}{$7^{+}$} & $\mathrm{F}$ & 55 & NS & $\begin{array}{l}\text { Vaxzevria } \\
\text { I dose }\end{array}$ & 6 days $\neq$ & $\begin{array}{c}\downarrow \text { PLTs, fibrinogen }+ \\
\text { anti-PF4 antibodies }+ \\
\text { SplVT }+ \text { AAT }+ \\
\text { intracerebral hemorrhage }\end{array}$ & $\mathrm{NP}$ & VITT & \multirow{6}{*}{ Possible } & \multirow{6}{*}{$\begin{array}{l}\text { Immune- } \\
\text { mediated }\end{array}$} \\
\hline & & $\mathrm{F}$ & 52 & NS & $\begin{array}{l}\text { Vaxzevria } \\
\text { I dose }\end{array}$ & 10 days $\neq$ & $\begin{array}{l}\downarrow \text { PLTs, fibrinogen }+ \\
\text { anti-PF4 antibodies }\end{array}$ & $\begin{array}{l}\text { Multiple organs } \\
\text { small vessels } \\
\text { thrombosis + CVST+ } \\
\text { intracerebral } \\
\text { hemorrhage }\end{array}$ & VITT & & \\
\hline & & M & 38 & NS & $\begin{array}{l}\text { Vaxzevria } \\
\text { I dose }\end{array}$ & 14 days $\neq$ & $\begin{array}{c}\downarrow \text { PLTs, fibrinogen }+ \\
\text { anti-PF4 antibodies }+ \\
\text { massive PAE }\end{array}$ & $\mathrm{NP}$ & VITT & & \\
\hline & & M & 25 & NS & $\begin{array}{l}\text { Vaxzevria } \\
\text { I dose }\end{array}$ & 9 days $\neq$ & $\begin{array}{c}\downarrow \text { PLTs, fibrinogen }+ \\
\text { anti-PF4 antibodies }+ \\
\text { CVST }\end{array}$ & $\mathrm{NP}$ & VITT & & \\
\hline & & $\mathrm{F}$ & 22 & NS & $\begin{array}{l}\text { Vaxzevria } \\
\text { I dose }\end{array}$ & 10 days $\neq$ & $\begin{array}{c}\downarrow \text { PLTs + anti-PF4 } \\
\text { antibodies + CVST + } \\
\text { intracerebral hemorrhage }\end{array}$ & $\mathrm{NP}$ & VITT & & \\
\hline & & $\mathrm{F}$ & 32 & NS & $\begin{array}{l}\text { Vaxzevria } \\
\text { I dose }\end{array}$ & 12 days $\neq$ & $\begin{array}{c}\downarrow \text { PLTs, fibrinogen }+ \\
\text { anti-PF4 antibodies }+ \\
\text { CVST }\end{array}$ & $\mathrm{NP}$ & VITT & & \\
\hline $\begin{array}{l}\text { See et al. } \\
2021[35]\end{array}$ & $3^{+}$ & NS & NS & $\begin{array}{l}\text { Two were obese, } \\
\text { none had risk } \\
\text { factors for CVST }\end{array}$ & Janssen & NS & $\begin{array}{c}\downarrow \text { PLTs }+ \text { CVST }+ \\
\text { intracerebral hemorrhage }\end{array}$ & NS & VITT & Possible & $\begin{array}{l}\text { Immune- } \\
\text { mediated }\end{array}$ \\
\hline
\end{tabular}


Table 1. Cont.

\begin{tabular}{|c|c|c|c|c|c|c|c|c|c|c|}
\hline References & N. & Sex Age (yrs) & $\begin{array}{l}\text { Pre-Existing } \\
\text { Conditions }\end{array}$ & $\begin{array}{l}\text { Type of } \\
\text { Vaccine }\end{array}$ & $\begin{array}{c}\text { Vax- } \\
\text { Symptoms } \\
\text { Interval }\end{array}$ & $\begin{array}{l}\text { Clinical Manifestations/ } \\
\text { Ante-Mortem Findings }\end{array}$ & $\begin{array}{c}\text { Post-Mortem } \\
\text { and/or } \\
\text { Autoptic Findings }\end{array}$ & $\begin{array}{l}\text { Cause of } \\
\text { Death }\end{array}$ & $\begin{array}{l}\text { Causal } \\
\text { Relation- } \\
\text { ship? }\end{array}$ & $\begin{array}{c}\text { Hypothetical } \\
\text { Pathophysiology }\end{array}$ \\
\hline $\begin{array}{l}\text { Verma et al. } \\
2021[36]\end{array}$ & $1^{+}$ & M & NS & $\begin{array}{l}\text { Spikevax } \\
\text { II dose }\end{array}$ & 14 days $\neq$ & $\begin{array}{c}\text { Tachycardia + ST-segment } \\
\text { elevation + global } \\
\text { biventricular dysfunction } \\
+ \text { left ventricular } \\
\text { hypertrophy }\end{array}$ & $\begin{array}{c}\text { Myocardial } \\
\text { inflammatory } \\
\text { infiltrate } \\
\text { (macrophages }+\mathrm{T} \\
\text { cells + eosinophils }+ \\
\text { B cells) }\end{array}$ & $\begin{array}{l}\text { Fulminant } \\
\text { myocarditis }\end{array}$ & Possible & NS \\
\hline
\end{tabular}

Total: $19 \quad$ F:M Mean

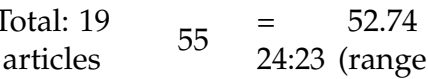

$£ \quad 22-91)^{£}$

${ }^{*}$ In this work, subjects' ages were not given for anonymization. ${ }^{+}$Only deceased subjects have been included in the table; the original paper counted more cases, but the other subjects survived. ${ }^{\S}$ In this study, age and sex of only one case (index case) were given. ${ }^{¥}$ Vaccination-death interval. $\neq$ Vaccination-hospital admission interval. ${ }^{£}$ The sex was specified in $47 / 55$ cases, the age in $43 / 55$ cases. 


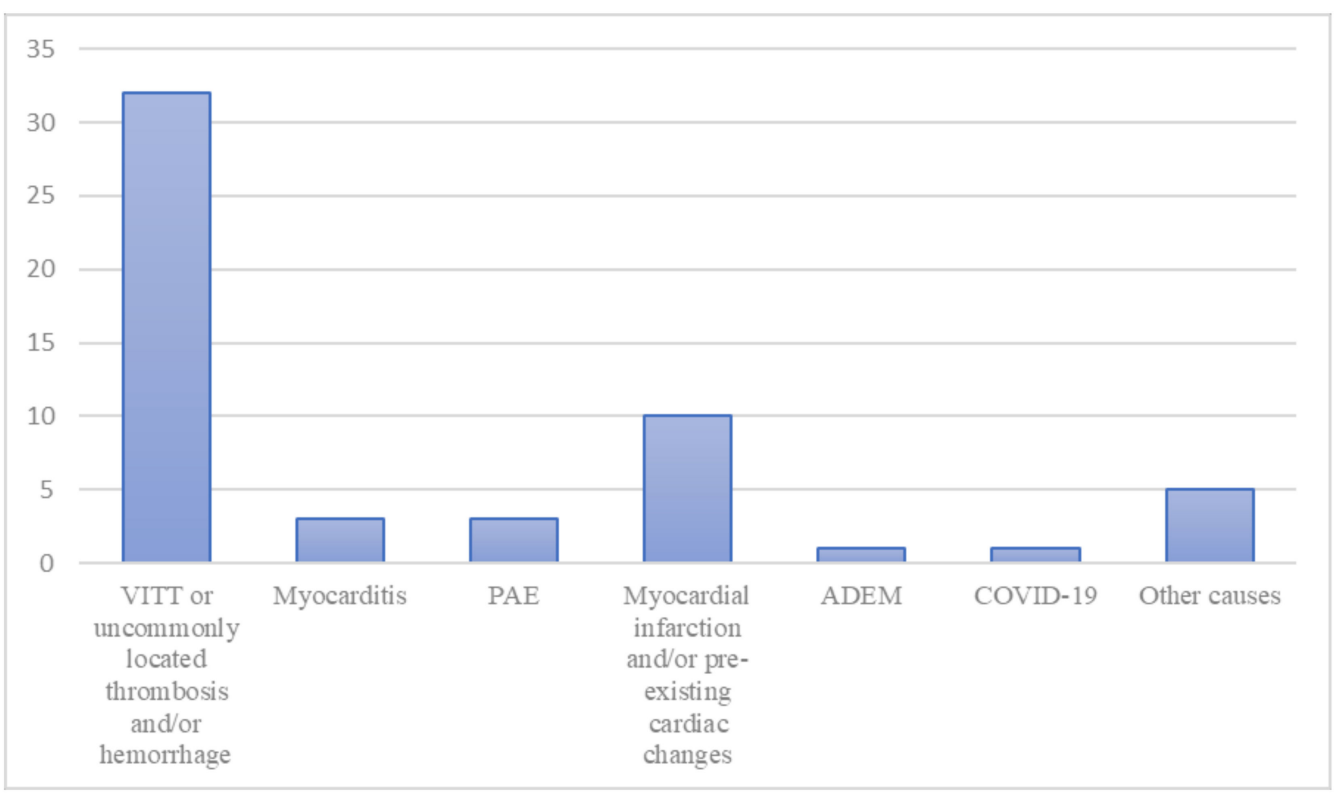

Figure 2. The distribution of the cause of death among the cases. "Other causes" includes one case of massive cerebral hemorrhage not associated with thrombosis or auto-antibodies, one case of anaphylactic reaction to anesthetics associated with cerebral venous sinus thrombosis and anti-PF4 antibodies, one case of hyperglycemic coma, one case of hemorrhagic shock due to aortic dissection and rupture, and one case of death due to the complications of rhabdomyolysis. ADEM indicates acute disseminated encephalomyelitis; COVID-19, coronavirus disease 2019; PAE, pulmonary embolism; VITT, vaccine-induced immune thrombotic thrombocytopenia.

The distribution concerning the type of vaccine was as follows: 35 cases of death following the Vaxzevria ${ }^{\circledR}(63.6 \%), 9$ cases following the Comirnaty ${ }^{\circledR}(16.4 \%), 6$ cases following the Spikevax ${ }^{\circledR}(10.9 \%)$, and 5 cases following the Janssen ${ }^{\circledR}(9.1 \%)$. Figure 3 illustrates the distribution of the type of vaccine among the 55 cases.

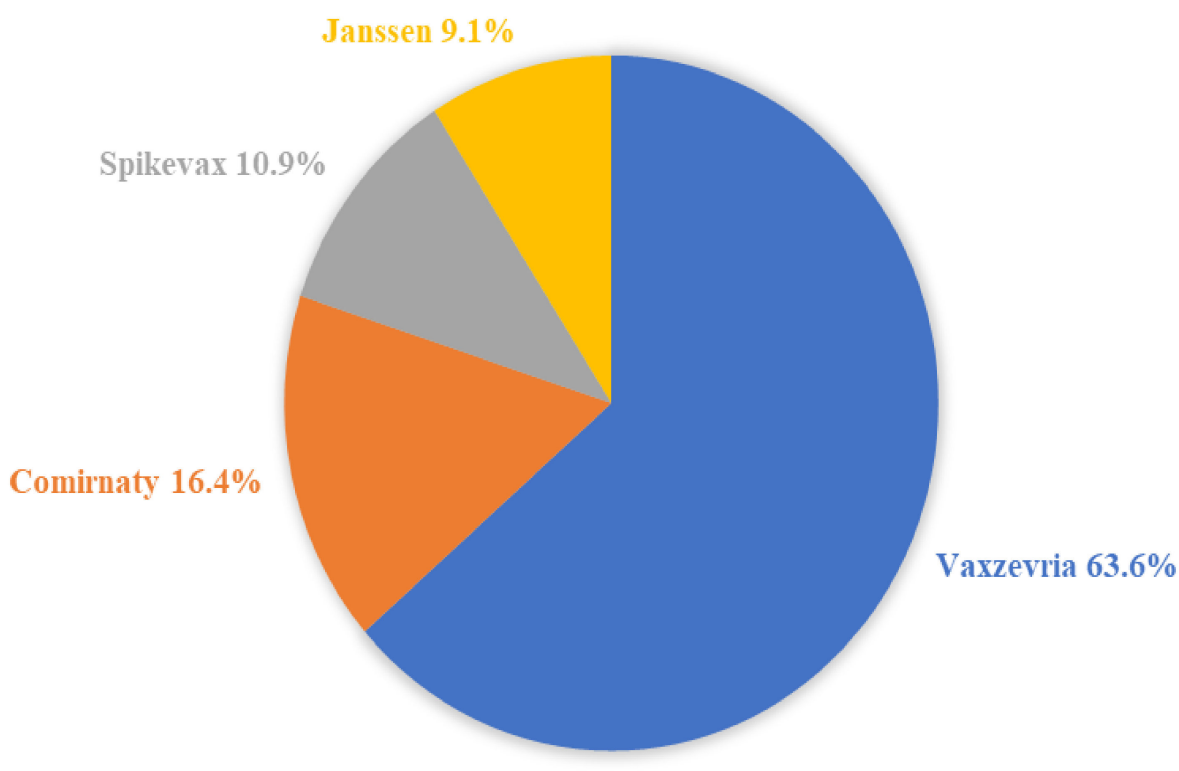

Figure 3. The distribution of the type of vaccine among the 55 cases of death after the vaccination.

The causal relationship between the death and the vaccine was not specified (meaning that the authors did not explicitly state if there was a causal relation or not) in eight cases $(14.5 \%)$, not demonstrated or improbable in 17 cases (30.9\%), possible in 15 cases $(27.3 \%)$, 
probable in one case $(1.8 \%)$, and demonstrated or very probable in 14 cases $(25.4 \%)$. Figure 4 shows the distribution of the probability of the causal relationship among the 55 cases.

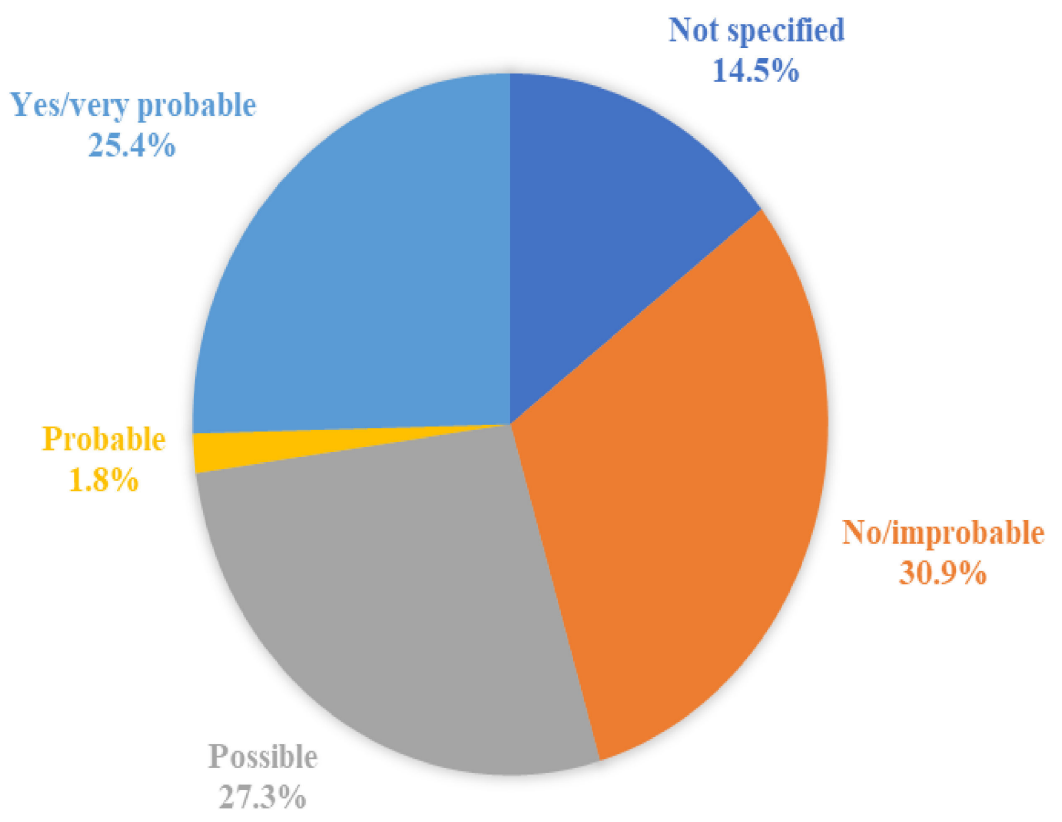

Figure 4. The distribution of the probability of the causal relationship among the 55 cases of death after the vaccination.

In Table 2, the distribution of causes of death per type of vaccine is shown. The cases in which the causal relationship between the vaccine and the death was not demonstrated or was improbable have not been included in this table.

Table 2. The distribution of causes of death per type of vaccine. The cases in which the causal relationship between the vaccine and the death was not demonstrated or improbable have not been included in this table, so the total number of cases that have been considered is 38. ADEM indicates acute disseminated encephalomyelitis; VITT, vaccine-induced immune thrombotic thrombocytopenia.

\begin{tabular}{|c|c|c|}
\hline Vaccine & Causes of Death & $\begin{array}{c}\text { N. Cases } \\
(\text { Total }=38)\end{array}$ \\
\hline $\begin{array}{c}\text { ChAdOx1 nCoV-19 } \\
\text { (Vaxzevria }^{\circledR}, \text { AstraZeneca) }\end{array}$ & $\begin{array}{c}\text { VITT or uncommonly located thrombosis } \\
\text { and/or hemorrhage } \\
\text { ADEM }\end{array}$ & $\begin{array}{c}27(71.0 \%) \\
1(2.6 \%)\end{array}$ \\
\hline $\begin{array}{c}\text { BNT162b2 } \\
\left(\text { Comirnaty }{ }^{\circledR},\right. \\
\text { BioNTech/Pfizer) }\end{array}$ & Myocarditis & $2(5.3 \%)$ \\
\hline $\begin{array}{c}\text { mRNA1273 } \\
\left(\text { Spikevax }{ }^{\circledR}, \text { Moderna) }\right.\end{array}$ & $\begin{array}{c}\text { VITT } \\
\text { Myocarditis } \\
\text { Rhabdomyolysis (with complications) }\end{array}$ & $\begin{array}{l}1(2.6 \%) \\
1(2.6 \%) \\
1(2.6 \%)\end{array}$ \\
\hline $\begin{array}{c}\text { Ad26.COV2.S } \\
\text { (Janssen }^{\circledR} \text {, Johnson\&Johnson) }\end{array}$ & $\begin{array}{c}\text { VITT } \\
\text { Myocardial infarction }\end{array}$ & $\begin{array}{l}4(10.5 \%) \\
1(2.6 \%)\end{array}$ \\
\hline
\end{tabular}

\section{Discussion}

According to WHO, there have been more than 260 million cases of COVID-19 infection and more than 5 million deaths reported globally, underlining the importance of an effective vaccination program as a powerful weapon to counteract the virus [6]. At the time of writing this paper, the European Medical Agency (EMA) has approved four vaccines: BNT162b2 $\left(\right.$ Comirnaty ${ }^{\circledR}$, BioNTech/Pfizer, New York, NY, USA), mRNA1273 (Spikevax ${ }^{\circledR}$, Moderna, 
Cambridge, MA, USA), Ad26.COV2.S (Janssen ${ }^{\circledR}$, Johnson\&Johnson, New Brunswick, NJ, USA), and ChAdOx1 nCoV-19/AZD1222 (Vaxzevria ${ }^{\circledR}$, AstraZeneca, Cambridge, UK).

Comirnaty ${ }^{\circledR}$ and Spikevax ${ }^{\circledR}$ work by introducing a piece of mRNA that contains the instructions for synthesizing the Spike protein, a surface protein that acts as a key allowing the virus to enter the cells. Once the human cells produce the Spike protein, they rapidly break down the mRNA [37-39]. This is the first time an mRNA vaccine has been administered to the population.

Ad26.COV2.S and ChAdOx1 are viral vector vaccines. They use an adenovirus to deliver a DNA genetic sequence encoding for the Spike protein into the cells [40]. Adenoviruses have already been used as vaccine agents in other infectious diseases, such as the Ebola infection [41].

It is necessary to overcome COVID-19 vaccine hesitancy to achieve a high percentage of vaccinated people [42]. However, some people are procrastinating and rejecting the vaccine because of the fear of death due to its side effects. They claim that these vaccines are new and therefore have not been studied enough to be safe [43-49]. Indeed, COVID-19 vaccines may cause side effects, as any other drug. The most common are mild and short-lived, such as fever, administration-site pain, weakness, etc. [50,51]. In addition, some severe adverse reactions have occurred. Regarding the mRNA vaccines, myocarditis, pericarditis, and anaphylaxis have been described, but these cases are very rare [52,53]. Moreover, it has been demonstrated that the viral vector vaccines can cause venous thrombosis associated with thrombocytopenia, so-called vaccine-induced immune thrombotic thrombocytopenia (VITT), and very few cases of Guillain-Barré syndrome have been reported [54-56].

We found 55 cases of death temporally related to COVID-19 vaccine administration. The male/female ratio was close to one (1.04), showing no difference between sexes. The ratio between people aged 50 years or younger and older than 50 years was 0.8 . So, it seems there is a slight predominance of older people among the cases. This is quite interesting, as we would have expected a more pronounced difference. Some possible reasons are that the death of a young person arouses greater interest, inducing the performance of investigations, and is more likely to be reported.

As mentioned before, more than eight billion doses of COVID-19 vaccines have been administered worldwide. However, searching the literature, we found only 55 cases of death temporally correlated with vaccination, and in 17 of these a causal relationship has been excluded. Therefore, these 17 cases of death are only temporally, and not causally, related to the vaccine administrations. Among the remaining 38 cases, in eight the probability of a causal correlation between the vaccine and the death was not specified, in 16 the authors stated that it was possible or probable, while in 14 cases the causal relationship was very probable/confirmed. The causes of death which can be considered vaccine-related were the following: VITT (27 cases with ChAdOx1, 4 cases with Ad26.COV2.S, and 1 case with mRNA1273), myocarditis (two cases with BNT162b2, one case with mRNA1273), acute disseminated encephalomyelitis (ADEM, one case with ChAdOx1), myocardial infarction (one case with Ad26.COV2.S), and complications of rhabdomyolysis (one case with mRNA1273). The ratio of deaths to the total number of administered vaccine doses (55 vs. millions of doses) clearly shows how rare fatal adverse events are, as has been found for other vaccines (for example, quadrivalent live attenuated and trivalent inactivated influenza vaccines) [57-59]. Moreover, the effectiveness of the vaccination programs in preventing hospital admission and COVID-19-related deaths has already been demonstrated $[60,61]$. Vaccines are the principal preventive strategy. Moreover, recent studies have shown the efficacy of new therapies in controlling the disease: monoclonal antibodies and antiviral drugs (e.g., molnupiravir, nirmatrelvir/ritonavir) [62]. In the future, the development of more effective vaccines (e.g., sterilizing immunity), the high percentage of vaccinated people - also in the developing countries—and effective therapeutic protocol will make COVID-19 a disease to live with [63].

A brief discussion about the assessment of the link between the vaccines and the deaths (causal or only temporal) is provided below. 


\subsection{Vaccine-Induced Immune Thrombotic Thrombocytopenia}

VITT is characterized by moderate to severe thrombocytopenia associated with venous and/or arterial thrombosis mainly in unusual locations, such as the cerebral venous sinus or the splanchnic veins [64]. The serum of the patients affected by VITT contains autoantibodies against the platelet factor 4 (PF4) antigen that can be identified by PF4 enzyme-linked immunosorbent assay (ELISA) [65]. VITT features resemble those of autoimmune heparin-induced thrombocytopenia (HIT) $[25,33,66]$. The production of PFA autoantibodies induces platelet activation through the formation of PF4-polyanion complexes, causing thrombocytopenia, disseminated intravascular coagulation, and atypically located thrombotic events. Even if the reason why the vaccine triggers the production of autoantibodies has not been disclosed yet, the causal relationship between VITT and adenoviral vector COVID-19 vaccines is confirmed [67]. Interestingly, we found a case of VITT related to an mRNA vaccine [31]. Even if this case is anecdotal, it suggests that VITT is not only related to adenoviral vectors and that other factors are implicated in its pathophysiology.

To confirm VITT in suspected cases, several recommendations have been published and diagnosis is based on anti-PF4 antibodies testing $[68,69]$. Therefore, the causal relationship between the death and the vaccine in such cases is quite easy to demonstrate or exclude.

\subsection{Myocarditis}

Myocarditis is a myocardium inflammation with myofiber damage and necrosis [70,71]. It is caused by various events, e.g., viral infections, drugs, etc., and it can lead to severe symptoms and death, even in young people [72,73]. Myocarditis has been reported as a side effect of vaccinations, such as influenza, hepatitis B, etc. [74]. So, it is not surprising that COVID-19 vaccines could also cause myocarditis. We found three cases of death from mRNA vaccine-related myocarditis. Cases of non-fatal myocarditis and pericarditis have also been reported [75-81]. Indeed, EMA warned about the risk of myocarditis and pericarditis with COVID-19 mRNA vaccines [52]. Moreover, the CDC Advisory Committee on Immunization Practices recognized that COVID-19 mRNA vaccines can cause myocarditis [82]. However, such events are rare, and even COVID-19 itself can cause myocarditis and pericarditis [83-86]. Therefore, the vaccine's benefits overcome the risks [87]. When a case of myocarditis or pericarditis occurs after a vaccination and without an alternative cause, a causal link with the vaccine is generally considered to be present or at least possible, even if a specific diagnostic test or laboratory analysis is not available $[88,89]$. Ante-mortem and post-mortem investigations can demonstrate features of myocarditis (for example, histologically) but cannot differentiate its etiology.

\subsection{Acute Disseminated Encephalomyelitis}

ADEM is an inflammation of the central nervous system associated with demyelination $[90,91]$. The clinical trials performed to evaluate the safety of the COVID-19 vaccines before general administration did not highlight cases of ADEM [92,93]. However, the AstraZeneca initial trial was provisionally stopped due to two cases of transverse myelitis that eventually were not considered to be undoubtedly related to the vaccine [94]. In the case of death due to ADEM after Vaxzeria administration reported in this review, the pathological mechanism that induced the encephalomyelitis was not clear and the authors did not state with certainty that it was causally related to the vaccine [28]. Another case of neuroinflammatory disorder after a COVID-19 mRNA vaccine (Comirnaty) has been published and some cases of ADEM have been reported in some international and national side effects reporting systems, such as the Vaccine Adverse Event Reporting System (VAERS) and EudraVigilance $[95,96]$. In such situations, given the fact that only anecdotal cases are reported in the literature, it is not possible to demonstrate a causal link with the vaccine, even in the absence of alternative causes. The risk of ADEM or other severe neurological disorders seems to be extremely low with COVID-19 vaccines. Vice versa, 
COVID-19 patients can manifest neuropathological features due to the infection, so the balance between risks and benefits is in favour of the vaccine [97].

\subsection{Rhabdomyolysis}

Rhabdomyolysis is due to the damage of the muscle that induces the release of intracellular myoglobin into the blood [98]. The consequent accumulation of myoglobin in the kidneys causes tubular necrosis and acute renal failure [99,100]. In the case of death after a COVID-19 vaccine (mRNA1273) described by Ajmera, the patient was hospitalized because of rhabdomyolysis, developed pneumonia and other complications, and then died [18]. Post-mortem investigations were not performed. Other cases of rhabdomyolysis after COVID-19 vaccination have been reported, both in the literature and in the side effects reporting systems (e.g., VAERS) [101-109]. Again, it is difficult to assess whether the link between the rhabdomyolysis is causal or only temporal and the diagnosis of vaccine-induced rhabdomyolysis is based on the exclusion of other causes.

\subsection{Myocardial Infarction}

The commonest cause of acute myocardial infarction is coronary artery occlusion due to the rupture and/or erosion of a vulnerable plaque, with consequent thrombus formation [110-116]. During the COVID-19 outbreak, there has been a reduction of cases of patients presenting with myocardial infarction $[117,118]$. However, even some cases of myocardial infarction induced by SARS-CoV-2 infection have been described [119]. The underlying pathological mechanism may involve acute plaque alterations induced by inflammation and cytokines [120-122]. Concerning COVID-19 vaccines, we found only one case of death due to acute myocardial infarction possibly related to Janssen vaccine administration [32]. Actually, the case is about a 69-year-old man who developed CVS thrombosis with anti-PF4 autoantibodies alongside coronary thrombosis. Myocardial histology showed fresh myocardial ischemia, while the cerebral tissue did not show any significant alteration. The authors attributed the death to acute myocardial infarction. The man died at home, so clinical information is not available. This is a peculiar case because the patient could also have been diagnosed with VITT, even if it was not the cause of death. The coronary thrombosis may also be related to the pro-coagulation state induced by the antiPF4 autoantibodies. Indeed, antiPF4/heparin antibodies seem to be an independent predictor of myocardial infarction in patients with acute coronary syndrome, suggesting that it may be involved somehow in the pathophysiology of such disease [123].

In any event, we must be clear that in our review we found other cases of myocardial infarction, both acute and recurrent, after COVID-19 vaccines, but in such cases the authors did not state there was a causal link. The relationship was considered to be only temporal. Edler et al. described the case of an old man with several comorbidities who was found dead at home two days after receiving a first dose of Comirnaty ${ }^{\circledR}$ (see Table 1) [23]. The autopsy revealed features of peripheral pulmonary embolism and recurrent myocardial infarction. Schneider and colleagues reported three cases [32]. The first is that of an obese 34-year-old woman who was found dead at home the day after receiving a first dose of Vaxzevria ${ }^{\circledR}$. Fresh myocardial ischemia along with myocardial scars and hypertrophy were the main autoptic findings. The second case regards a 57-year-old man who died two days after Vaxzeria ${ }^{\circledR}$ administration (unknown dose). Again, the autopsy revealed cardiac hypertrophy, myocardial scars, coronary sclerosis, and a fresh myocardial infarction. The third case is that of a 72-year-old woman who died in the vaccination center soon after Comirnaty ${ }^{\circledR}$ administration. The first hypothesis was an anaphylactic reaction, but the autopsy showed coronary sclerosis, myocardial scars, coronary thrombosis, and acute myocardial infarction, while the anaphylaxis diagnostics were negative. In these cases, it was not possible to establish a causal relationship between the vaccine and acute myocardial ischemia, so the causal relationship was not determined.

The relatively high frequency of death and sudden death due to myocardial infarction and the high number of administered COVID-19 vaccine doses each day may suggest 
that there is only a casual and chronological relationship between the two events. In any case, we think further studies are needed to deepen this topic. We suggest in such cases to perform the VITT diagnostics and to look for anti-PF4 autoantibodies, as in the case previously described, to verify if they could be involved in myocardial infarction after the COVID-19 vaccination.

A limit of our study is the small sample size and the fact that the cases collected in this review do not represent the whole number of deaths after COVID-19 vaccine. We think there could be a sort of "reporting bias" concerning this type of event. At first, researchers could be inhibited from reporting cases of vaccine-related death because of the fear of being held up as "no-vax". In addition, we noticed that authors tended to be prudent in stating whether or not there was a causal correlation between a vaccine and a death. In fact, in eight cases the causal relationship was not explicitly established. Therefore, post-mortem investigations should be considered essential since the autoptic data and histological analysis could provide more information about the pathological features in such cases of death.

\section{Conclusions}

Given the current pandemic situation, it is fundamental that most people get vaccinated. Concerns about vaccines' side effects and consequent hesitancy are serious factors that slow down the immunization campaign; thus, the scientific community needs to be cohesive in maintaining that vaccination is fundamental to containing the spread of SARSCoV-2 [124-127]. However, it is just as important to evaluate the safety of these vaccines and continuously monitor potential side effects. In this study, we examined 55 cases of death following COVID-19 vaccination reported in the literature to analyze all the available data on fatal cases and assess the existence of a possible correlation with vaccine administration. Given the small number of severe adverse reactions and deaths reported, it is beyond doubt that the benefits of vaccination outweigh the risks. Nevertheless, we think more studies are needed to deepen understanding of possible vaccine-related pathophysiological mechanisms, and researchers should report such cases. Furthermore, we want to encourage post-mortem investigations in cases of death following COVID-19 vaccination because they are essential to better clarify whether a causal relationship between vaccination and death does exist.

Author Contributions: Conceptualization, A.M. and M.D.P.; study design, A.C.M.; validation, M.D.P. and E.T.; literature search, A.B. and A.M. data collection and analysis, A.C.M. and A.B.; writingoriginal draft preparation, A.C.M. and A.M.; figures and tables, A.C.M.; writing-review and editing, E.T., P.F. and V.F.; supervision, V.F. All authors have read and agreed to the published version of the manuscript.

Funding: This research received no external funding.

Institutional Review Board Statement: Not applicable.

Informed Consent Statement: Not applicable.

Data Availability Statement: Not applicable.

Conflicts of Interest: The authors declare no conflict of interest.

\section{References}

1. Calatayud, A.; Bedoya-Maya, F.; Sánchez González, S.; Giraldez, F. Containing the spatial spread of COVID-19 through the trucking network. Transp. Policy (Oxf.) 2022, 115, 4-13. [CrossRef] [PubMed]

2. Lau, H.; Khosrawipour, V.; Kocbach, P.; Mikolajczyk, A.; Schubert, J.; Bania, J.; Khosrawipour, T. The positive impact of lockdown in Wuhan on containing the COVID-19 outbreak in China. J. Travel Med. 2020, 18, 27. [CrossRef] [PubMed]

3. Kohli, M.; Maschio, M.; Becker, D.; Weinstein, M.C. The potential public health and economic value of a hypothetical COVID-19 vaccine in the United States: Use of cost-effectiveness modeling to inform vaccination prioritization. Vaccine 2021, 39, 1157-1164. [CrossRef] [PubMed] 
4. World Health Organization (WHO). COVID-19 Vaccines. Available online: https://www.who.int/emergencies/diseases/novelcoronavirus-2019/ covid-19-vaccines (accessed on 11 December 2021).

5. Centers for Disease Control and Prevention (CDC). Immunization: The Basics. Available online: https://www.cdc.gov/vaccines / vac-gen/imz-basics.htm (accessed on 11 December 2021).

6. World Health Organization (WHO). WHO Coronavirus (COVID-19) Dashboard-Data Table. Available online: https://covid19. who.int/table (accessed on 11 December 2021).

7. World Health Organization (WHO). WHO Coronavirus (COVID-19) Dashboard-Overview. Available online: https://covid19. who.int/ (accessed on 11 December 2021).

8. European Centre for Disease Prevention and Control (ECDC). COVID-19 Vaccine Tracker. Available online: https://vaccinetracker. ecdc.europa.eu/public/extensions/COVID-19/vaccine-tracker.html\#uptake-tab (accessed on 11 December 2021).

9. European Medicines Agency (EMA). European Medicines Agency EMA Recommends First COVID-19 Vaccine for Authorisation in the EU. 2020. Available online: https://www.ema.europa.eu/en/news/ema-recommends-first-covid-19-vaccineauthorisation-eu (accessed on 11 December 2021).

10. European Medicines Agency (EMA). EMA Recommends COVID-19 Vaccine Moderna for Authorisation in the EU. 2021. Available online: https:/ / www.ema.europa.eu/en/news/ema-recommends-covid-19-vaccine-moderna-authorisation-eu (accessed on 11 December 2021).

11. European Medicines Agency (EMA). EMA Recommends COVID-19 Vaccine AstraZeneca for Authorisation in the EU. 2021. Available online: https:/ / www.ema.europa.eu/en/news/ema-recommends-covid-19-vaccine-astrazeneca-authorisation-eu (accessed on 11 December 2021).

12. European Medicines Agency (EMA). EMA Recommends COVID-19 Vaccine Janssen for Authorisation in the EU. 2021. Available online: https:/ / www.ema.europa.eu/en/news/ema-recommends-covid-19-vaccine-janssen-authorisation-eu (accessed on 11 December 2021).

13. Chen, P.W.; Tsai, Z.Y.; Chao, T.H.; Li, Y.H.; Hou, C.J.; Liu, P.Y. Addressing Vaccine-Induced Immune Thrombotic Thrombocytopenia (VITT) Following COVID-19 Vaccination: A Mini-Review of Practical Strategies. Acta Cardiol. Sin. 2021, 37, 355-364. [PubMed]

14. Yan, Z.P.M.; Yang, M.; Lai, C.L. COVID-19 Vaccines: A Review of the Safety and Efficacy of Current Clinical Trials. Pharmaceuticals 2021, 14, 406. [CrossRef]

15. World Health Organization (WHO). Manufacturing, Safety and Quality Control of Vaccines. Available online: https://www.who. int/news-room/feature-stories/detail/manufacturing-safety-and-quality-control (accessed on 11 December 2021).

16. Sadarangani, M.; Abu Raya, B.; Conway, J.M.; Iyaniwura, S.A.; Falcao, R.C.; Colijn, C.; Coombs, D.; Gantt, S. Importance of COVID-19 vaccine efficacy in older age groups. Vaccine 2021, 39, 2020-2023. [CrossRef]

17. Liberati, A.; Altman, D.G.; Tetzlaff, J.; Mulrow, C.; Gøtzsche, P.C.; Ioannidis, J.P.; Clarke, M.; Devereaux, P.J.; Kleijnen, J.; Moher, D. The PRISMA statement for reporting systematic reviews and meta-analyses of studies that evaluate healthcare interventions: Explanation and elaboration. BMJ 2009, 339, b2700. [CrossRef]

18. Ajmera, K.M. Fatal Case of Rhabdomyolysis Post-COVID-19 Vaccine. Infect. Drug Resist. 2021, 14, 3929-3935. [CrossRef]

19. Bjørnstad-Tuveng, T.H.; Rudjord, A.; Anker, P. Fatal cerebral haemorrhage after COVID-19 vaccine. Tidsskr. Nor. Laegeforen. 2021, 141,1-4.

20. Castelli, G.P.; Pognani, C.; Sozzi, C.; Franchini, M.; Vivona, L. Cerebral venous sinus thrombosis associated with thrombocytopenia post-vaccination for COVID-19. Crit. Care 2021, 25, 137. [CrossRef]

21. Choi, S.; Lee, S.; Seo, J.W.; Kim, M.J.; Jeon, Y.H.; Park, J.H.; Lee, J.K.; Yeo, N.S. Myocarditis-induced Sudden Death after BNT162b2 mRNA COVID-19 Vaccination in Korea: Case Report Focusing on Histopathological Findings. J. Korean Med. Sci. 2021, 36 , e286. [CrossRef] [PubMed]

22. D’Agostino, V.; Caranci, F.; Negro, A.; Piscitelli, V.; Tuccillo, B.; Fasano, F.; Sirabella, G.; Marano, I.; Granata, V.; Grassi, R.; et al. A Rare Case of Cerebral Venous Thrombosis and Disseminated Intravascular Coagulation Temporally Associated to the COVID-19 Vaccine Administration. J. Pers. Med. 2021, 11, 285. [CrossRef] [PubMed]

23. Edler, C.; Klein, A.; Schröder, A.S.; Sperhake, J.P.; Ondruschka, B. Deaths associated with newly launched SARS-CoV-2 vaccination (Comirnaty ${ }^{\circledR}$ ). Leg. Med. (Tokyo) 2021, 51, 101895. [CrossRef] [PubMed]

24. Franchini, M.; Testa, S.; Pezzo, M.; Glingani, C.; Caruso, B.; Terenziani, I.; Pognani, C.; Bellometti, S.A.; Castelli, G. Cerebral venous thrombosis and thrombocytopenia post-COVID-19 vaccination. Thromb. Res. 2021, 202, 182-183. [CrossRef] [PubMed]

25. Greinacher, A.; Thiele, T.; Warkentin, T.E.; Weisser, K.; Kyrle, P.A.; Eichinger, S. Thrombotic Thrombocytopenia after ChAdOx1 nCov-19 Vaccination. N. Engl. J. Med. 2021, 384, 2092-2101. [CrossRef] [PubMed]

26. Jamme, M.; Mosnino, E.; Hayon, J.; Franchineau, G. Fatal cerebral venous sinus thrombosis after COVID-19 vaccination. Intensive Care Med. 2021, 47, 790-791. [CrossRef]

27. Mehta, P.R.; Apap Mangion, S.; Benger, M.; Stanton, B.R.; Czuprynska, J.; Arya, R.; Sztriha, L.K. Cerebral venous sinus thrombosis and thrombocytopenia after COVID-19 vaccination-A report of two UK cases. Brain Behav. Immun. 2021, 95, 514-517. [CrossRef]

28. Permezel, F.; Borojevic, B.; Lau, S.; de Boer, H.H. Acute disseminated encephalomyelitis (ADEM) following recent Oxford/AstraZeneca COVID-19 vaccination. Forensic. Sci. Med. Pathol. 2021, 1-6, epub ahead of print. [CrossRef]

29. Pomara, C.; Sessa, F.; Ciaccio, M.; Dieli, F.; Esposito, M.; Garozzo, S.F.; Giarratano, A.; Prati, D.; Rappa, F.; Salerno, M.; et al. Post-mortem findings in vaccine-induced thrombotic thombocytopenia. Haematologica 2021, 106, 2291-2293. [CrossRef] 
30. Rodriguez, E.V.C.; Bouazza, F.Z.; Dauby, N.; Mullier, F.; d'Otreppe, S.; Jissendi Tchofo, P.; Bartiaux, M.; Sirjacques, C.; Roman, A.; Hermans, C.; et al. Fatal vaccine-induced immune thrombotic thrombocytopenia (VITT) post Ad26.COV2.S: First documented case outside US. Infection 2021, 1-6, epub ahead of print. [CrossRef]

31. Sangli, S.; Virani, A.; Cheronis, N.; Vannatter, B.; Minich, C.; Noronha, S.; Bhagavatula, R.; Speredelozzi, D.; Sareen, M.; Kaplan, R.B. Thrombosis With Thrombocytopenia After the Messenger RNA-1273 Vaccine. Ann. Intern. Med. 2021, 174, 1480-1482. [CrossRef] [PubMed]

32. Schneider, J.; Sottmann, L.; Greinacher, A.; Hagen, M.; Kasper, H.U.; Kuhnen, C.; Schlepper, S.; Schmidt, S.; Schulz, R.; Thiele, T.; et al. Postmortem investigation of fatalities following vaccination with COVID-19 vaccines. Int. J. Legal Med. 2021, 135, 2335-2345. [CrossRef] [PubMed]

33. Schultz, N.H.; Sørvoll, I.H.; Michelsen, A.E.; Munthe, L.A.; Lund-Johansen, F.; Ahlen, M.T.; Wiedmann, M.; Aamodt, A.H.; Skattør, T.H.; Tjønnfjord, G.E.; et al. Thrombosis and Thrombocytopenia after ChAdOx1 nCoV-19 Vaccination. N. Engl. J. Med. 2021, 384, 2124-2130. [CrossRef] [PubMed]

34. Scully, M.; Singh, D.; Lown, R.; Poles, A.; Solomon, T.; Levi, M.; Goldblatt, D.; Kotoucek, P.; Thomas, W.; Lester, W. Pathologic Antibodies to Platelet Factor 4 after ChAdOx1 nCoV-19 Vaccination. N. Engl. J. Med. 2021, 384, 2202-2211. [CrossRef] [PubMed]

35. See, I.; Su, J.R.; Lale, A.; Woo, E.J.; Guh, A.Y.; Shimabukuro, T.T.; Streiff, M.B.; Rao, A.K.; Wheeler, A.P.; Beavers, S.F.; et al. US Case Reports of Cerebral Venous Sinus Thrombosis with Thrombocytopenia after Ad26.COV2.S Vaccination, March 2 to April 21, 2021. JAMA 2021, 325, 2448-2456. [CrossRef]

36. Verma, A.K.; Lavine, K.J.; Lin, C.Y. Myocarditis after Covid-19 mRNA Vaccination. N. Engl. J. Med. 2021, 385, 1332-1334. [CrossRef]

37. Verbeke, R.; Lentacker, I.; De Smedt, S.C.; Dewitte, H. The dawn of mRNA vaccines: The COVID-19 case. J. Control. Release 2021, 333, 511-520. [CrossRef]

38. Machado, B.A.S.; Hodel, K.V.S.; Fonseca, L.M.D.S.; Mascarenhas, L.A.B.; Andrade, L.P.C.D.S.; Rocha, V.P.C.; Soares, M.B.P.; Berglund, P.; Duthie, M.S.; Reed, S.G.; et al. The Importance of RNA-Based Vaccines in the Fight against COVID-19: An Overview. Vaccines 2021, 9, 1345. [CrossRef]

39. Sadarangani, M.; Marchant, A.; Kollmann, T.R. Immunological mechanisms of vaccine-induced protection against COVID-19 in humans. Nat. Rev. Immunol. 2021, 21, 475-484. [CrossRef]

40. Mendonça, S.A.; Lorincz, R.; Boucher, P.; Curiel, D.T. Adenoviral vector vaccine platforms in the SARS-CoV-2 pandemic. NPJ Vaccines 2021, 6, 97. [CrossRef]

41. Choi, M.J.; Cossaboom, C.M.; Whitesell, A.N.; Dyal, J.W.; Joyce, A.; Morgan, R.L.; Campos-Outcalt, D.; Person, M.; Ervin, E.; Yu, Y.C.; et al. Use of Ebola Vaccine: Recommendations of the Advisory Committee on Immunization Practices, United States, 2020. MMWR Recomm. Rep. 2021, 70, 1-12. [CrossRef]

42. Matrajt, L.; Eaton, J.; Leung, T.; Brown, E.R. Vaccine optimization for COVID-19: Who to vaccinate first? Sci. Adv. 2021, 7, eabf1374. [CrossRef]

43. Rief, W. Fear of Adverse Effects and COVID-19 Vaccine Hesitancy: Recommendations of the Treatment Expectation Expert Group. JAMA Health Forum 2021, 2, e210804. [CrossRef]

44. MacDonald, N.E. SAGE Working Group on Vaccine Hesitancy. Vaccine hesitancy: Definition, scope and determinants. Vaccine 2015, 33, 4161-4164. [CrossRef] [PubMed]

45. Sirikalyanpaiboon, M.; Ousirimaneechai, K.; Phannajit, J.; Pitisuttithum, P.; Jantarabenjakul, W.; Chaiteerakij, R.; Paitoonpong, L. COVID-19 vaccine acceptance, hesitancy, and determinants among physicians in a university-based teaching hospital in Thailand. BMC Infect. Dis. 2021, 21, 1174. [CrossRef] [PubMed]

46. Urrunaga-Pastor, D.; Bendezu-Quispe, G.; Herrera-Añazco, P.; Uyen-Cateriano, A.; Toro-Huamanchumo, C.J.; RodriguezMorales, A.J.; Hernandez, A.V.; Benites-Zapata, V.A. Cross-sectional analysis of COVID-19 vaccine intention, perceptions and hesitancy across Latin America and the Caribbean. Travel Med. Infect. Dis. 2021, 41, 102059. [CrossRef] [PubMed]

47. Dror, A.A.; Eisenbach, N.; Taiber, S.; Morozov, N.G.; Mizrachi, M.; Zigron, A.; Srouji, S.; Sela, E. Vaccine hesitancy: The next challenge in the fight against COVID-19. Eur. J. Epidemiol. 2020, 35, 775-779. [CrossRef] [PubMed]

48. Puri, N.; Coomes, E.A.; Haghbayan, H.; Gunaratne, K. Social media and vaccine hesitancy: New updates for the era of COVID-19 and globalized infectious diseases. Hum. Vaccin. Immunother. 2020, 16, 2586-2593. [CrossRef] [PubMed]

49. Barello, S.; Nania, T.; Dellafiore, F.; Graffigna, G.; Caruso, R. 'Vaccine hesitancy' among university students in Italy during the COVID-19 pandemic. Eur. J. Epidemiol. 2020, 35, 781-783. [CrossRef]

50. Zhu, F.C.; Li, Y.H.; Guan, X.H.; Hou, L.H.; Wang, W.J.; Li, J.X.; Wu, S.P.; Wang, B.S.; Wang, Z.; Wang, L.; et al. Safety, tolerability, and immunogenicity of a recombinant adenovirus type- 5 vectored COVID-19 vaccine: A dose-escalation, open-label, non-randomised, first-in-human trial. Lancet 2020, 395, 1845-1854. [CrossRef]

51. European Medicines Agency (EMA). Safety of COVID-19 Vaccines. Available online: https://www.ema.europa.eu/en/humanregulatory / overview / public-health-threats / coronavirus-disease-covid-19/treatments-vaccines/vaccines-covid-19/safetycovid-19-vaccines (accessed on 11 December 2021).

52. COVID-19 mRNA Vaccines Comirnaty and Spikevax: Risk of Myocarditis and Pericarditis. 2021. Available online: https://www. ema.europa.eu/en/medicines/dhpc/covid-19-mrna-vaccines-comirnaty-spikevax-risk-myocarditis-pericarditis (accessed on 11 December 2021). 
53. Gargano, J.W.; Wallace, M.; Hadler, S.C.; Langley, G.; Su, J.R.; Oster, M.E.; Broder, K.R.; Gee, J.; Weintraub, E.; Shimabukuro, T.; et al. Use of mRNA COVID-19 Vaccine After Reports of Myocarditis Among Vaccine Recipients: Update from the Advisory Committee on Immunization Practices-United States, June 2021. MMWR Morb. Mortal. Wkly. Rep. 2021, 70, 977-982. [CrossRef] [PubMed]

54. Woo, E.J.; Mba-Jonas, A.; Dimova, R.B.; Alimchandani, M.; Zinderman, C.E.; Nair, N. Association of Receipt of the Ad26.COV2.S COVID-19 Vaccine With Presumptive Guillain-Barré Syndrome, February-July 2021. JAMA 2021, 326, 1606-1613. [CrossRef] [PubMed]

55. Aleem, A.; Nadeem, A.J. Coronavirus (COVID-19) Vaccine-Induced Immune Thrombotic Thrombocytopenia (VITT); [Updated 18 July 2021]; StatPearls Publishing: Treasure Island, FL, USA, 2021. Available online: https://www.ncbi.nlm.nih.gov/books/NBK57060 5/ (accessed on 12 December 2021).

56. Franchini, M.; Liumbruno, G.M.; Pezzo, M. COVID-19 vaccine-associated immune thrombosis and thrombocytopenia (VITT): Diagnostic and therapeutic recommendations for a new syndrome. Eur. J. Haematol. 2021, 107, 173-180. [CrossRef] [PubMed]

57. Vellozzi, C.; Burwen, D.R.; Dobardzic, A.; Ball, R.; Walton, K.; Haber, P. Safety of trivalent inactivated influenza vaccines in adults: Background for pandemic influenza vaccine safety monitoring. Vaccine 2009, 27, 2114-2120. [CrossRef]

58. Haber, P.; Moro, P.L.; Cano, M.; Lewis, P.; Stewart, B.; Shimabukuro, T.T. Post-licensure surveillance of quadrivalent live attenuated influenza vaccine United States, Vaccine Adverse Event Reporting System (VAERS), July 2013-June 2014. Vaccine 2015, 33, 1987-1992. [CrossRef]

59. Gidengil, C.; Goetz, M.B.; Newberry, S.; Maglione, M.; Hall, O.; Larkin, J.; Motala, A.; Hempel, S. Safety of vaccines used for routine immunization in the United States: An updated systematic review and meta-analysis. Vaccine 2021, 39, 3696-3716. [CrossRef]

60. COVID-19 Cases and Hospitalizations by COVID-19 Vaccination Status and Previous COVID-19 Diagnosis. Available online: https:/ / www.cdc.gov/mmwr/volumes/71/wr/mm7104e1.htm (accessed on 3 February 2022).

61. Jabłońska, K.; Aballéa, S.; Toumi, M. The real-life impact of vaccination on COVID-19 mortality in Europe and Israel. Public Health 2021, 198, 230-237. [CrossRef]

62. Parums, D.V. Editorial: Current Status of Oral Antiviral Drug Treatments for SARS-CoV-2 Infection in Non-Hospitalized Patients. Med. Sci. Monit. 2022, 28, e935952. [CrossRef]

63. Borio, L.L.; Bright, R.A.; Emanuel, E.J. A National Strategy for COVID-19 Medical Countermeasures: Vaccines and Therapeutics. JAMA 2022, 327, 215-216. [CrossRef]

64. Pavord, S.; Scully, M.; Hunt, B.J.; Lester, W.; Bagot, C.; Craven, B.; Rampotas, A.; Ambler, G.; Makris, M. Clinical Features of Vaccine-Induced Immune Thrombocytopenia and Thrombosis. N. Engl. J. Med. 2021, 385, 1680-1689. [CrossRef]

65. Matsuo, T.; Matsuo, M.; Sugimoto, T.; Wanaka, K. Anti-heparin/PF4 complexes by ELISA in patients with disseminated intravascular coagulation. Pathophysiol. Haemost. Thromb. 2007, 36, 305-310. [CrossRef]

66. Lee, G.M.; Arepally, G.M. Heparin-induced thrombocytopenia. Hematol. Am. Soc. Hematol. Educ. Program. 2013, 2013, 668-674. [CrossRef]

67. McGonagle, D.; De Marco, G.; Bridgewood, C. Mechanisms of Immunothrombosis in Vaccine-Induced Thrombotic Thrombocytopenia (VITT) Compared to Natural SARS-CoV-2 Infection. J. Autoimmun. 2021, 121, 102662. [CrossRef] [PubMed]

68. Nazy, I.; Sachs, U.J.; Arnold, D.M.; McKenzie, S.E.; Choi, P.; Althaus, K.; Ahlen, M.T.; Sharma, R.; Grace, R.F.; Bakchoul, T. Recommendations for the clinical and laboratory diagnosis of VITT against COVID-19: Communication from the ISTH SSC Subcommittee on Platelet Immunology. J. Thromb. Haemost. 2021, 19, 1585-1588. [CrossRef] [PubMed]

69. Platton, S.; Bartlett, A.; MacCallum, P.; Makris, M.; McDonald, V.; Singh, D.; Scully, M.; Pavord, S. Evaluation of laboratory assays for anti-platelet factor 4 antibodies after ChAdOx1 nCOV-19 vaccination. J. Thromb. Haemost. 2021, 19, 2007-2013. [CrossRef]

70. Cooper, L.T., Jr. Myocarditis. N. Engl. J. Med. 2009, 360, 1526-1538. [CrossRef] [PubMed]

71. Pomara, C.; Villani, A.; D’Errico, S.; Riezzo, I.; Turillazzi, E.; Fineschi, V. Acute myocarditis mimicking acute myocardial infarction: A clinical nightmare with forensic implications. Int. J. Cardiol. 2006, 112, 119-121. [CrossRef] [PubMed]

72. $\quad$ Lynge, T.H.; Nielsen, T.S.; Gregers Winkel, B.; Tfelt-Hansen, J.; Banner, J. Sudden cardiac death caused by myocarditis in persons aged 1-49 years: A nationwide study of 14294 deaths in Denmark. Forensic. Sci. Res. 2019, 4, 247-256. [CrossRef]

73. Sagar, S.; Liu, P.P.; Cooper, L.T., Jr. Myocarditis. Lancet 2012, 379, 738-747. [CrossRef]

74. Su, J.R.; McNeil, M.M.; Welsh, K.J.; Marquez, P.L.; Ng, C.; Yan, M.; Cano, M.V. Myopericarditis after vaccination, Vaccine Adverse Event Reporting System (VAERS), 1990-2018. Vaccine 2021, 39, 839-845. [CrossRef]

75. Umei, T.C.; Kishino, Y.; Shiraishi, Y.; Inohara, T.; Yuasa, S.; Fukuda, K. Recurrence of myopericarditis following mRNA COVID-19 vaccination in a male adolescent. CJC Open 2021. epub ahead of print. [CrossRef] [PubMed]

76. Miqdad, M.A.; Nasser, H.; Alshehri, A.; Mourad, A.R. Acute Myocarditis Following the Administration of the Second BNT162b2 COVID-19 Vaccine Dose. Cureus 2021, 13, e18880. [CrossRef]

77. Das, B.B.; Kohli, U.; Ramachandran, P.; Nguyen, H.H.; Greil, G.; Hussain, T.; Tandon, A.; Kane, C.; Avula, S.; Duru, C.; et al. Myopericarditis after messenger RNA Coronavirus Disease 2019 Vaccination in Adolescents 12 to 18 Years of Age. J. Pediatr. 2021, 238, 26-32.e1. [CrossRef] [PubMed]

78. Patel, Y.R.; Louis, D.W.; Atalay, M.; Agarwal, S.; Shah, N.R. Cardiovascular magnetic resonance findings in young adult patients with acute myocarditis following mRNA COVID-19 vaccination: A case series. J. Cardiovasc. Magn. Reson. 2021, 23, 101. [CrossRef] 
79. Patrignani, A.; Schicchi, N.; Calcagnoli, F.; Falchetti, E.; Ciampani, N.; Argalia, G.; Mariani, A. Acute myocarditis following Comirnaty vaccination in a healthy man with previous SARS-CoV-2 infection. Radiol. Case Rep. 2021, 16, 3321-3325. [CrossRef] [PubMed]

80. Hajra, A.; Gupta, M.; Ghosh, B.; Ashish, K.; Patel, N.; Manek, G.; Rai, D.; Bandyopadhyay, D.; Lavie, C.J. Proposed Pathogenesis, Characteristics, and Management of COVID-19 mRNA Vaccine-Related Myopericarditis. Am. J. Cardiovasc. Drugs 2021, 24, 9-26. [CrossRef] [PubMed]

81. Facetti, S.; Giraldi, M.; Vecchi, A.L.; Rogiani, S.; Nassiacos, D. Miocardite acuta in giovane adulto due giorni dopo vaccino Pfizer [Acute myocarditis in a young adult two days after Pfizer vaccination]. G. Ital. Cardiol. (Rome) 2021, 22, 891-893. (In Italian)

82. Centers for Disease Control and Prevention (CDC). Advisory Committee on Immunization Practices (ACIP). Coronavirus Disease 2019 (COVID-19) Vaccines. Available online: https:/ /www.cdc.gov/vaccines/acip/meetings/slides-2021-06.html (accessed on 11 December 2021).

83. Paul, J.F.; Charles, P.; Richaud, C.; Caussin, C.; Diakov, C. Myocarditis revealing COVID-19 infection in a young patient. Eur Heart J. Cardiovasc. Imaging 2020, 21, 776. [CrossRef]

84. Siripanthong, B.; Nazarian, S.; Muser, D.; Deo, R.; Santangeli, P.; Khanji, M.Y.; Cooper, L.T., Jr.; Chahal, C.A.A. Recognizing COVID-19-related myocarditis: The possible pathophysiology and proposed guideline for diagnosis and management. Heart Rhythm 2020, 17, 1463-1471. [CrossRef]

85. Maiese, A.; Frati, P.; Del Duca, F.; Santoro, P.; Manetti, A.C.; La Russa, R.; Di Paolo, M.; Turillazzi, E.; Fineschi, V. Myocardial Pathology in COVID-19-Associated Cardiac Injury: A Systematic Review. Diagnostics 2021, 11, 1647. [CrossRef]

86. Patone, M.; Mei, X.W.; Handunnetthi, L.; Dixon, S.; Zaccardi, F.; Shankar-Hari, M.; Watkinson, P.; Khunti, K.; Harnden, A.; Coupland, C.A.C.; et al. Risks of myocarditis, pericarditis, and cardiac arrhythmias associated with COVID-19 vaccination or SARS-CoV-2 infection. Nat. Med. 2021. epub ahead of print. [CrossRef] [PubMed]

87. Bozkurt, B.; Kamat, I.; Hotez, P.J. Myocarditis with COVID-19 mRNA Vaccines. Circulation 2021, 144, 471-484. [CrossRef] [PubMed]

88. Sciaccaluga, C.; D’Ascenzi, F.; Cameli, M.; Gallotta, M.; Menci, D.; Antonelli, G.; Banchi, B.; Mochi, V.; Valente, S.; Focardi, M. Acute myopericarditis after mRNA COVID-19 vaccine. Eur. Heart J. Suppl. 2021, 23 (Suppl. G), suab142-049. [CrossRef]

89. Das, B.B.; Moskowitz, W.B.; Taylor, M.B.; Palmer, A. Myocarditis and Pericarditis Following mRNA COVID-19 Vaccination: What Do We Know So Far? Children 2021, 8, 607. [CrossRef]

90. Pohl, D.; Alper, G.; Van Haren, K.; Kornberg, A.J.; Lucchinetti, C.F.; Tenembaum, S.; Belman, A.L. Acute disseminated encephalomyelitis: Updates on an inflammatory CNS syndrome. Neurology 2016, 87 (Suppl. 2), S38-S45. [CrossRef]

91. Tenembaum, S.; Chitnis, T.; Ness, J.; Hahn, J.S.; International Pediatric MS Study Group. Acute disseminated encephalomyelitis. Neurology 2007, 68 (Suppl. 2), S23-S36. [CrossRef]

92. Baden, L.R.; El Sahly, H.M.; Essink, B.; Kotloff, K.; Frey, S.; Novak, R.; Diemert, D.; Spector, S.A.; Rouphael, N.; Creech, C.B.; et al. Efficacy and Safety of the mRNA-1273 SARS-CoV-2 Vaccine. N. Engl. J. Med. 2021, 384, 403-416. [CrossRef]

93. Polack, F.P.; Thomas, S.J.; Kitchin, N.; Absalon, J.; Gurtman, A.; Lockhart, S.; Perez, J.L.; Pérez Marc, G.; Moreira, E.D.; Zerbini, C.; et al. Safety and Efficacy of the BNT162b2 mRNA Covid-19 Vaccine. N. Engl. J. Med. 2020, 383, 2603-2615. [CrossRef]

94. Knoll, M.D.; Wonodi, C. Oxford-AstraZeneca COVID-19 vaccine efficacy. Lancet 2021, 397, 72-74. [CrossRef]

95. Vogrig, A.; Janes, F.; Gigli, G.L.; Curcio, F.; Negro, I.D.; D'Agostini, S.; Fabris, M.; Valente, M. Acute disseminated encephalomyelitis after SARS-CoV-2 vaccination. Clin. Neurol. Neurosurg. 2021, 208, 106839. [CrossRef]

96. Goss, A.L.; Samudralwar, R.D.; Das, R.R.; Nath, A. ANA Investigates: Neurological Complications of COVID-19 Vaccines. Ann. Neurol. 2021, 89, 856-857. [CrossRef]

97. Maiese, A.; Manetti, A.C.; Bosetti, C.; Del Duca, F.; La Russa, R.; Frati, P.; Di Paolo, M.; Turillazzi, E.; Fineschi, V. SARS-CoV-2 and the brain: A review of the current knowledge on neuropathology in COVID-19. Brain Pathol. 2021, 31, e13013. [CrossRef] [PubMed]

98. Cabral, B.M.I.; Edding, S.N.; Portocarrero, J.P.; Lerma, E.V. Rhabdomyolysis. Dis. Mon. 2020, 66, 101015. [CrossRef] [PubMed]

99. Bosch, X.; Poch, E.; Grau, J.M. Rhabdomyolysis and acute kidney injury. N. Engl. J. Med. 2009, 361, 62-72, Erratum in N. Engl. J. Med. 2011, 364, 1982. [CrossRef]

100. Heyne, N.; Guthoff, M.; Weisel, K.C. Rhabdomyolysis and acute kidney injury. N. Engl. J. Med. 2009, 361, 1412. [PubMed]

101. Nassar, M.; Chung, H.; Dhayaparan, Y.; Nyein, A.; Acevedo, B.J.; Chicos, C.; Zheng, D.; Barras, M.; Mohamed, M.; Alfishawy, M.; et al. COVID-19 vaccine induced rhabdomyolysis: Case report with literature review. Diabetes Metab. Syndr. 2021, 15, 102170. [CrossRef] [PubMed]

102. Gelbenegger, G.; Cacioppo, F.; Firbas, C.; Jilma, B. Rhabdomyolysis Following Ad26.COV2.S COVID-19 Vaccination. Vaccines 2021, 9, 956. [CrossRef]

103. Mack, M.; Nichols, L.; Guerrero, D.M. Rhabdomyolysis Secondary to COVID-19 Vaccination. Cureus 2021, 13, e15004. [CrossRef]

104. Jin, M.; Tong, Q. Rhabdomyolysis as Potential Late Complication Associated with COVID-19. Emerg. Infect. Dis. 2020, 26, 1618-1620. [CrossRef]

105. Singh, B.; Kaur, P.; Reid, R.R. Case Reports: Rhabdomyolysis Associated with COVID-19. Am. Fam. Physician 2020, 102, 645-648.

106. Faissner, S.; Richter, D.; Ceylan, U.; Schneider-Gold, C.; Gold, R. COVID-19 mRNA vaccine induced rhabdomyolysis and fasciitis. J. Neurol. 2021, 25, 1-2. [CrossRef] [PubMed] 
107. Elias, C.; Cardoso, P.; Gonçalves, D.; Vaz, I.; Cardoso, L. Rhabdomyolysis Following Administration of Comirnaty ${ }^{\circledR}$. Eur. J. Case Rep. Intern. Med. 2021, 8, 002796. [CrossRef] [PubMed]

108. Tan, A.; Stepien, K.M.; Narayana, S.T.K. Carnitine palmitoyltransferase II deficiency and post-COVID vaccination rhabdomyolysis. QJM 2021, 114, 596-597. [CrossRef]

109. Hakroush, S.; Tampe, B. Case Report: ANCA-Associated Vasculitis Presenting With Rhabdomyolysis and Pauci-Immune Crescentic Glomerulonephritis after Pfizer-BioNTech COVID-19 mRNA Vaccination. Front. Immunol. 2021, 12, 762006. [CrossRef]

110. Falk, E.; Shah, P.K.; Fuster, V. Coronary plaque disruption. Circulation 1995, 92, 657-671. [CrossRef]

111. Neri, M.; Fineschi, V.; Di Paolo, M.; Pomara, C.; Riezzo, I.; Turillazzi, E.; Cerretani, D. Cardiac oxidative stress and inflammatory cytokines response after myocardial infarction. Curr. Vasc. Pharmacol. 2015, 13, 26-36. [CrossRef]

112. Neri, M.; Riezzo, I.; Pascale, N.; Pomara, C.; Turillazzi, E. Ischemia/Reperfusion Injury following Acute Myocardial Infarction: A Critical Issue for Clinicians and Forensic Pathologists. Mediators. Inflamm. 2017, 2017, 7018393. [CrossRef] [PubMed]

113. Manetti, A.C.; Maiese, A.; Paolo, M. D; De Matteis, A; La Russa, R.; Turillazzi, E.; Frati, P.; Fineschi, V. MicroRNAs and Sepsis-Induced Cardiac Dysfunction: A Systematic Review. Int. J. Mol. Sci. 2020, 22, 321. [CrossRef] [PubMed]

114. Turillazzi, E.; Di Paolo, M.; Neri, M.; Riezzo, I.; Fineschi, V. A theoretical timeline for myocardial infarction: Immunohistochemical evaluation and western blot quantification for Interleukin-15 and Monocyte chemotactic protein-1 as very early markers. J. Transl. Med. 2014, 12, 188. [CrossRef]

115. Turillazzi, E.; Pomara, C.; Bello, S.; Neri, M.; Riezzo, I.; Fineschi, V. The meaning of different forms of structural myocardial injury, immune response and timing of infarct necrosis and cardiac repair. Curr. Vasc. Pharmacol. 2015, 13, 6-19. [CrossRef]

116. Shah, P.K. Plaque disruption and coronary thrombosis: New insight into pathogenesis and prevention. Clin. Cardiol. 1997, 20 (Suppl. 2), II-38-II-44. [CrossRef]

117. Solomon, M.D.; McNulty, E.J.; Rana, J.S.; Leong, T.K.; Lee, C.; Sung, S.H.; Ambrosy, A.P.; Sidney, S.; Go, A.S. The Covid-19 Pandemic and the Incidence of Acute Myocardial Infarction. N. Engl. J. Med. 2020, 383, 691-693. [CrossRef] [PubMed]

118. De Rosa, S.; Spaccarotella, C.; Basso, C.; Calabrò, M.P.; Curcio, A.; Filardi, P.P.; Mancone, M.; Mercuro, G.; Muscoli, S.; Nodari, S.; et al. Reduction of hospitalizations for myocardial infarction in Italy in the COVID-19 era. Eur. Heart J. 2020, 41, 2083-2088, Erratum in Eur. Heart J. 2021, 42, 683Erratum in Eur. Heart J. 2021, 42, 322. [CrossRef] [PubMed]

119. Capaccione, K.M.; Leb, J.S.; D'souza, B.; Utukuri, P.; Salvatore, M.M. Acute myocardial infarction secondary to COVID-19 infection: A case report and review of the literature. Clin. Imaging 2021, 72, 178-182. [CrossRef]

120. Modin, D.; Claggett, B.; Sindet-Pedersen, C.; Lassen, M.C.H.; Skaarup, K.G.; Jensen, J.U.S.; Fralick, M.; Schou, M.; Lamberts, M.; Gerds, T.; et al. Acute COVID-19 and the Incidence of Ischemic Stroke and Acute Myocardial Infarction. Circulation 2020, 142, 2080-2082. [CrossRef]

121. Maiese, A.; Passaro, G.; De Matteis, A.; Fazio, V.; La Russa, R.; Di Paolo, M. Thromboinflammatory response in SARS-CoV-2 sepsis. Med. Leg. J. 2020, 88, 78-80. [CrossRef]

122. Zanza, C.; Racca, F.; Longhitano, Y.; Piccioni, A.; Franceschi, F.; Artico, M.; Abenavoli, L.; Maiese, A.; Passaro, G.; Volonnino, G.; et al. Risk Management and Treatment of Coagulation Disorders Related to COVID-19 Infection. Int. J. Environ. Res. Public Health 2021, 18, 1268. [CrossRef]

123. Williams, R.T.; Damaraju, L.V.; Mascelli, M.A.; Barnathan, E.S.; Califf, R.M.; Simoons, M.L.; Deliargyris, E.N.; Sane, D.C. Antiplatelet factor 4/heparin antibodies: An independent predictor of 30-day myocardial infarction after acute coronary ischemic syndromes. Circulation 2003, 107, 2307-2312. [CrossRef]

124. D'Errico, S.; Turillazzi, E.; Zanon, M.; Viola, R.V.; Frati, P.; Fineschi, V. The Model of "Informed Refusal" for Vaccination: How to Fight against Anti-Vaccinationist Misinformation without Disregarding the Principle of Self-Determination. Vaccines 2021, 9, 110. [CrossRef]

125. D’Errico, S.; Zanon, M.; Concato, M.; Peruch, M.; Scopetti, M.; Frati, P.; Fineschi, V. "First Do No Harm". No-Fault Compensation Program for COVID-19 Vaccines as Feasibility and Wisdom of a Policy Instrument to Mitigate Vaccine Hesitancy. Vaccines 2021, 9 , 1116. [CrossRef]

126. Di Fazio, N.; Delogu, G.; Bertozzi, G.; Fineschi, V.; Frati, P. SARS-CoV2 Vaccination Adverse Events Trend in Italy: A Retrospective Interpretation of the Last Year (December 2020-September 2021). Vaccines 2022, 10, 216. [CrossRef]

127. Maiese, A.; Russa, R.; Santoro, P.; Matteis, A.; Paolo, M.D. Future litigation after Covid-19 pandemic in Italy. Med. Leg. J. 2021, 89, 148-149. [CrossRef] [PubMed] 